\title{
ROBUSTNESS IN THE GRAPH TOPOLOGY OF A COMMON ADAPTIVE CONTROLLER*
}

\author{
MARK FRENCH ${ }^{\dagger}$, ACHIM ILCHMANN ${ }^{\ddagger}$, AND EUGENE P. RYAN ${ }^{\S}$
}

\begin{abstract}
For any $m$-input, $m$-output, finite-dimensional, linear, minimum-phase plant $P$ with first Markov parameter having spectrum in the open right-half complex plane, it is well known that the adaptive output feedback control $C$, given by $u=-k y, \dot{k}=\|y\|^{2}$, yields a closed-loop system $[P, C]$ for which the state converges to zero, the signal $k$ converges to a finite limit, and all other signals are of class $L^{2}$. It is first shown that these properties continue to hold in the presence of $L^{2}$-input and $L^{2}$-output disturbances. By establishing gain function stability of an appropriate closed-loop operator, it is proved that these properties also persist when the plant $P$ is replaced by a stabilizable and detectable linear plant $P_{1}$ within a sufficiently small neighbourhood of $P$ in the graph topology, provided that the plant initial data and the $L^{2}$ magnitude of the disturbances are sufficiently small. Example 9 of Georgiou \& Smith (IEEE Trans. Autom. Control 42(9) 1200-1221, 1997) is revisited. Unstable behaviour for large initial conditions and/or large $L^{2}$ disturbances is shown, demonstrating that the bounds obtained from the $L^{2}$ theory are qualitatively tight: this contrasts with the $L^{\infty}$ robustness analysis of Georgiou \& Smith which is insufficiently tight to predict the stable behaviour for small initial conditions and zero disturbances.
\end{abstract}

Keywords: adaptive control, gap metric, robust stability.

1. Introduction. In an important paper in 1997, the well-established concept of the gap metric for linear systems [9] was extended to a nonlinear setting by Georgiou and Smith [3]. The central property analysed in the nonlinear gap framework is that of robust stability, i.e. the property that, if $\mathcal{W}$ is some requisite class (for example, $L^{\infty}$ or $L^{2}$ ) to which the signals of a nominal closed-loop plant/controller configuration belong, then the closed-loop signals remain in $\mathcal{W}$ if the nominal plant is replaced by another plant which is sufficiently close in the gap sense. Gain function stability (a concept made precise in Sub-section 2.3) of the closed-loop operator mapping external disturbances to the input and output of the nominal plant provides a sufficient condition for robust stability (however, in contrast with the results in the linear setting, gain function stability is not a necessary condition for robust stability in the nonlinear setting). The nonlinear gap framework has been used to investigate the robustness (or lack of robustness) of certain classical adaptive controllers and variants thereof.

1. Working in an $L^{\infty}$ setting, Example 9 of [3] (see also [4]) considers the controller (ubiquitous in the adaptive control literature)

$$
u=-k y, \quad \dot{k}=y^{2}
$$

applied to the scalar linear plant $\dot{y}=a y+u$, for some $a \in \mathbb{R}$, and shows that the closed-loop operator mapping external disturbances onto the input and output of the nominal plant is not gain function stable. Whilst the lack of gain function stability does not preclude robust stability, numerical and other informal evidence was presented which suggested that, with non-zero initial conditions, the closed-loop

\footnotetext{
* This research was supported by Deutsche Forschungsgemeinschaft, grant IL 25/3-1

${ }^{\dagger}$ School of Electronics and Computer Science, University of Southampton, Southampton SO17 1BJ, UK, mcf@ecs.soton.ac.uk

‡Institut für Mathematik, Technische Universität Ilmenau, Weimarer Straße 25, 98693 Ilmenau, DE, ilchmann@mathematik.tu-ilmenau.de

$\S$ Department of Mathematical Sciences, University of Bath, Claverton Down, Bath BA2 7AY, UK, epr@maths.bath.ac.uk
} 
system is not robustly stable, even in the absence of disturbances. One consequence of the results of the present paper is to clarify the latter suggestion: we prove that, in the absence of disturbances, the closed-loop system is - with sufficiently small initial data - robustly stable but fails to be robustly stable for large initial data.

2. In [1], the nonlinear gap framework is used in an $L^{2}$ setting to establish robust stability properties of the controller $u=-k^{\frac{1}{4}} y, \dot{k}=y^{2}$ when applied to (a) singleinput, single-output, linear, minimum-phase, relative-degree-one, nominal plants with positive high-frequency gain, and (b) a class of perturbed plants, where the gap metric distance between the nominal and perturbed plants is constrained by a function of the norms of the external disturbances.

The present paper shows that the analysis developed in [1] can also be applied to the more familiar adaptive controller (1.1) (and its multivariable counterpart). This is considered to be important as such controllers form the basis for many adaptive designs see e.g. $[5,6]$. In particular, in an $L^{2}$ setting, we establish a robust stability result for nominal $m$-input, $m$-output, finite-dimensional, stabilizable and detectable linear plants $(A, B, C)$ which are minimum phase and are such that the first Markov parameter $C B$ has its spectrum in the open right-half complex plane (we denote the class of such plants by $\mathcal{M}$ ). With reference to Figure 1.1, in the absence of external disturbances (that is, with $u_{0}=0=y_{0}$ ), it is well known that, for every plant in $\mathcal{M}$ and all initial plant/controller data $\left(x^{0}, k^{0}\right) \in \mathbb{R}^{n} \times \mathbb{R}_{+}\left(\mathbb{R}_{+}:=[0, \infty)\right)$, the closed loop is such that (i) $u_{1}, y_{1} \in L^{2}\left(\mathbb{R}_{+}, \mathbb{R}^{m}\right)$, (ii) $x(t) \rightarrow 0$ as $t \rightarrow \infty$, and (iii) $k(t) \rightarrow k^{\infty} \in \mathbb{R}_{+}$as $t \rightarrow \infty$. First, we show that properties (i)-(iii) persist

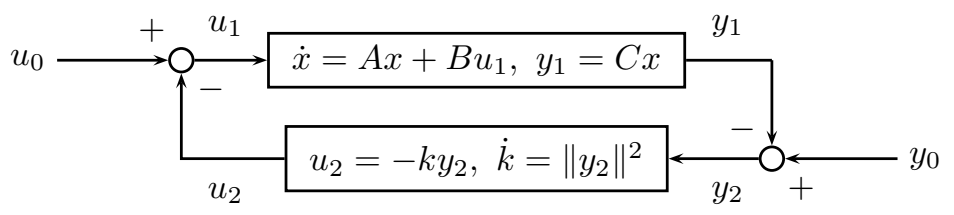

FIG. 1.1. The adaptive closed-loop system.

under external disturbances $u_{0}, y_{0} \in L^{2}\left(\mathbb{R}_{+}, \mathbb{R}^{m}\right)$. Secondly, we consider the question of robust stability of the closed-loop with respect to both external $L^{2}$ disturbances and perturbations of the plant $(A, B, C)$ : to what extent do the above properties (i)-(iii) persist if $(A, B, C) \in \mathcal{M}$ is perturbed to another $m$-input, $m$-output, linear, finite-dimensional, stabilizable and detectable plant $\left(A_{p}, B_{p}, C_{p}\right) \notin \mathcal{M}$ ?

An appropriate conceptual framework in which to pose and answer such questions is provided by the gap metric. We show that properties (i)-(iii) persist if $(A, B, C)$ and $\left(A_{p}, B_{p}, C_{p}\right)$ are sufficiently close in the gap metric. The associated bounds on the robust stability margin have a semi-global nature insofar as they depend on the "size" of the external disturbances and initial data.

In the case of zero initial conditions, the linear gap metric $\delta_{0}$ measures the size of the smallest stable co-prime factor perturbation between plants. Thus the stability results of the present paper have a interpretation within the framework of linear robust control where co-prime factor perturbations form the widely accepted model for unstructured uncertainties. For purposes of illustration, one expression for the linear gap $\delta_{0}$ is given in the frequency domain as follows. Let $\left(A_{1}, B_{1}, C_{1}\right)$ and $\left(A_{2}, B_{2}, C_{2}\right)$ be single-input, single-output stabilizable linear systems with respective transfer functions $\hat{P}_{1}$ and $\hat{P}_{2}$. Then $\hat{P}_{1}$ and $\hat{P}_{2}$ admit normalized right co-prime factorizations over 
$R H^{\infty}$, the class of rational functions that are analytic and bounded on the open half plane $\mathbb{C}_{+}:=\{\lambda \in \mathbb{C} \mid \operatorname{Re}(\lambda)>0\}$. In particular, there exist $N_{i}, D_{i} \in R H^{\infty}$ such that

$$
\hat{P}_{i}=N_{i} D_{i}^{-1}, \quad N_{i}^{*} N_{i}+D_{i}^{*} D_{i}=1, \quad i=1,2 .
$$

For $(i, j)=(1,2),(2,1)$, define the directed gap

$\vec{\delta}_{0}\left(\hat{P}_{i}, \hat{P}_{j}\right):=\inf \left\{\left\|\left(\Delta_{N}, \Delta_{D}\right)\right\|_{H^{\infty}} \mid \Delta_{N}, \Delta_{D} \in R H^{\infty}, \hat{P}_{j}=\left(N_{i}+\Delta_{N}\right)\left(D_{i}+\Delta_{D}\right)^{-1}\right\}$,

(with the convention inf $\emptyset:=+\infty$ ). The linear gap between $\hat{P}_{1}$ and $\hat{P}_{2}$ is given by

$$
\delta_{0}\left(\hat{P}_{1}, \hat{P}_{2}\right) \equiv \delta_{0}\left(\hat{P}_{2}, \hat{P}_{1}\right)=\max \left\{\vec{\delta}_{0}\left(\hat{P}_{1}, \hat{P}_{2}\right), \vec{\delta}_{0}\left(\hat{P}_{2}, \hat{P}_{1}\right)\right\} .
$$

\begin{tabular}{|c|c|c|c|c|}
\hline & $\hat{P}_{1}(s)$ & $\hat{P}_{2}(s)$ & & Reference \\
\hline i) & $\frac{1}{s-\theta}$ & $\frac{|\lambda|^{2}}{(s-\lambda)(s-\bar{\lambda})(s-\theta)}$ & $\operatorname{Re}(\lambda) \leq-\varepsilon^{-1}$ & {$[1]$} \\
\hline ii) & $\frac{1}{s-\theta}$ & $\frac{N(M-s)}{(N+s)(M+s)(s-\theta)}$ & $N, M \geq \varepsilon^{-1}$ & $\S 3.4$, Example 3.10 \\
\hline iii) & $\frac{1}{s-\theta}$ & $\frac{(M-s)}{(M+s)(s-\theta)}$ & $M \geq \varepsilon^{-1}$ & $\S 4.5$, see also $[3]$ \\
\hline
\end{tabular}

We remark that the gap between the following plants $\hat{P}_{1}$ and $\hat{P}_{2}$ tends to zero as $\varepsilon \rightarrow 0$ :

Example i) is the classical Rohrs' example [7] which first drew the attention of the adaptive control community to the robustness issue. As observed in [1], Example ii) is of particular interest since $\hat{P}_{2}$ exhibits none of the classical assumptions of adaptive control: in particular, the sign of the high frequency gain and the relative degree of $\hat{P}_{2}$ differ from those of the nominal plant $\hat{P}_{1}$ and, moreover, $\hat{P}_{2}$ is not minimum phase. Example ii) is considered in more detail in Section 3.4. Example iii) is comprised of an all-pass factor in series with the nominal plant and is considered extensively in Section 4. Example iii) also coincides with Example 9 in [3] to which our general $L^{2}$ theory applies to conclude robust stability provided the initial data and $L^{2}$ disturbance norms are sufficiently small. In Section 4 , we additionally prove the lack of robustness when the initial data or the $L^{2}$ disturbances are large. Moreover, we clarify some of the informal arguments in the $L^{\infty}$ setting of [3].

2. Background concepts and terminology. The material in this section is based on [3, Section II] and [1, Section 2].

2.1. Preliminaries. Whilst our goal is to establish stability of various configurations of plant and controller, the nonlinear nature of the controller is such that finite-time blow up of solutions of the closed-loop system cannot be ruled out a priori. To accommodate the potential for such a behaviour in the analysis, we introduce the following artifacts. Let $\mathcal{X}$ be a nonempty set and, for $0<\omega \leq \infty$, let $\mathcal{S}_{\omega}$ denote the set of locally integrable maps $[0, \omega) \rightarrow \mathcal{X}$. For simplicity, we write $\mathcal{S}:=\mathcal{S}_{\infty}$. For $0<\tau<\omega \leq \infty, T_{\tau}: \mathcal{S}_{\omega} \rightarrow \mathcal{S}$ denotes the operator given by

$$
T_{\tau} v:= \begin{cases}v(t), & t \in[0, \tau) \\ 0, & \text { otherwise. }\end{cases}
$$

With $\mathcal{V} \subset \mathcal{S}$ we associate spaces as follows: $\mathcal{V}_{e}=\left\{v \in \mathcal{S} \mid T_{\tau} v \in \mathcal{V} \forall \tau>0\right\}$, the extended space; $\mathcal{V}_{\omega}=\left\{v \in \mathcal{S}_{\omega} \mid T_{\tau} v \in \mathcal{V} \forall \tau \in(0, \omega)\right\}, 0<\omega \leq \infty ; \mathcal{V}_{a}=\cup_{\omega \in(0, \infty]} \mathcal{V}_{\omega}$, 
the ambient space. If $v, w \in \mathcal{V}_{a}$ with $\left.v\right|_{I}=\left.w\right|_{I}$ on $I=\operatorname{dom}(v) \cap \operatorname{dom}(w)$, then we write $v=w$. Note that $\mathcal{V} \subset \mathcal{V}_{e} \subset \mathcal{V}_{a}$ are strict inclusions and $\mathcal{V}_{\infty}=\mathcal{V}_{e}$

For $(f, g) \in \mathcal{V}_{a} \times \mathcal{V}_{a}$, the domains of $f$ and $g$ may be different; we adopt the convention $\operatorname{dom}(f, g):=\operatorname{dom}(f) \cap \operatorname{dom}(g)$.

We define $\mathcal{V} \subset \mathcal{S}$ to be a signal space if, and only if, it is a vector space. In our applications, frequently $\mathcal{V}$ will be a normed signal space, such as $L^{p}\left(\mathbb{R}_{+}, \mathbb{R}^{m}\right)$ for $1 \leq p \leq \infty$, in which case, $\mathcal{V}_{e}=L_{\text {loc }}^{p}\left(\mathbb{R}_{+}, \mathbb{R}^{m}\right), \mathcal{V}_{\omega}=L_{\text {loc }}^{p}\left([0, \omega), \mathbb{R}^{m}\right)$ for $\omega \in(0, \infty]$ and $\mathcal{V}_{a}=\cup_{0<\omega \leq \infty} L_{\text {loc }}^{p}\left([0, \omega), \mathbb{R}^{m}\right)$. It is important to note that $\mathcal{V}_{\omega} \neq L^{p}\left([0, \omega), \mathbb{R}^{m}\right)$. Throughout the paper we consider only those normed signal space $\mathcal{V}$ which have the property that $\sup _{\tau \geq 0}\left\|T_{\tau} x\right\|<\infty$ implies $x \in \mathcal{V}$. We observe that $L^{p}\left(\mathbb{R}_{+}, \mathbb{R}^{m}\right)$ for $1 \leq p \leq \infty$ is such a normed signal space. We will often write $\|x\|_{\tau}=\left\|T_{\tau} x\right\|$.

For a normed signal space $\mathcal{U}$ and a Euclidean space $\mathbb{R}^{n}$, we will also consider subsets of spaces of the form $\mathcal{V}=\mathbb{R}^{n} \times \mathcal{U}$, which, on identifying each $\theta \in \mathbb{R}^{n}$ with the constant signal $t \mapsto \theta$, can be thought of as a normed signal space with norm given by $\|(\theta, x)\|=\sqrt{|\theta|^{2}+\|x\|_{\mathcal{U}}^{2}}$.

2.2. Well posedness. A mapping $Q: \mathcal{X}_{1} \rightarrow \mathcal{X}_{2}$ between signal spaces is said to be causal if, and only if, for all $\tau>0, x, y \in \mathcal{X}_{1}, T_{\tau} x=T_{\tau} y$ implies $T_{\tau} Q x=T_{\tau} Q y$. Let $\mathcal{U}$ and $\mathcal{Y}$ be normed signal spaces and let $P: \mathcal{U}_{a} \rightarrow \mathcal{Y}_{a}$ and $C: \mathcal{Y}_{a} \rightarrow \mathcal{U}_{a}$ be causal mappings representing the plant and controller, respectively. Our central concern is the system of equations

$$
[P, C]: \quad y_{1}=P u_{1}, \quad u_{2}=C y_{2}, \quad u_{0}=u_{1}+u_{2}, \quad y_{0}=y_{1}+y_{2}
$$

corresponding to the closed-loop feedback configuration as depicted in Figure 2.1.

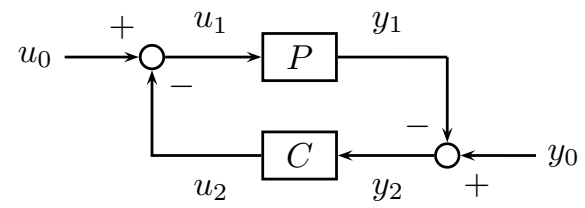

FIG. 2.1. The closed-loop system $[P, C]$.

By a solution of (2.1) we mean the following. For $w_{0}=\left(u_{0}, y_{0}\right) \in \mathcal{W}:=\mathcal{U} \times \mathcal{Y}$, a pair $\left(w_{1}, w_{2}\right)=\left(\left(u_{1}, y_{1}\right),\left(u_{2}, y_{2}\right)\right) \in \mathcal{W}_{a} \times \mathcal{W}_{a}, \mathcal{W}_{a}:=\mathcal{U}_{a} \times \mathcal{Y}_{a}$, is a solution of $(2.1)$ if, and only if, (2.1) holds on $\operatorname{dom}\left(w_{1}, w_{2}\right)$. The (possibly empty) set of all solutions is denoted by $\mathcal{X}_{w_{0}}:=\left\{\left(w_{1}, w_{2}\right) \in \mathcal{W}_{a} \times \mathcal{W}_{a} \mid\left(w_{1}, w_{2}\right)\right.$ solves (2.1) $\}$. The closed-loop system $[P, C]$, given by $(2.1)$, is said have: (a) the existence property if, and only if, $\mathcal{X}_{w_{0}} \neq \emptyset ;(\mathrm{b})$ the uniqueness property if, and only if, for each $w_{0} \in \mathcal{W}$,

$$
\begin{aligned}
&\left(\hat{w}_{1}, \hat{w}_{2}\right),\left(\tilde{w}_{1}, \tilde{w}_{2}\right) \in \mathcal{X}_{w_{0}} \\
& \Longrightarrow\left(\hat{w}_{1}, \hat{w}_{2}\right)=\left(\tilde{w}_{1}, \tilde{w}_{2}\right) \quad \text { on } \operatorname{dom}\left(\hat{w}_{1}, \hat{w}_{2}\right) \cap \operatorname{dom}\left(\tilde{w}_{1}, \tilde{w}_{2}\right) .
\end{aligned}
$$

Assume that $[P, C]$ has the existence and uniqueness properties. For each $w_{0} \in \mathcal{W}$, define $\omega_{w_{0}}, 0<\omega_{w_{0}} \leq \infty$, by the property $\left[0, \omega_{w_{0}}\right):=\cup_{\left(\hat{w}_{1}, \hat{w}_{2}\right) \in \mathcal{X}_{w_{0}}} \operatorname{dom}\left(\hat{w}_{1}, \hat{w}_{2}\right)$ and define $\left(w_{1}, w_{2}\right) \in \mathcal{W}_{a} \times \mathcal{W}_{a}$, with $\operatorname{dom}\left(w_{1}, w_{2}\right)=\left[0, \omega_{w_{0}}\right)$, by the property $\left.\left(w_{1}, w_{2}\right)\right|_{[0, t)} \in \mathcal{X}_{w_{0}}$ for all $t \in\left[0, \omega_{w_{0}}\right)$. This construction induces an operator $H_{P, C}: \mathcal{W} \rightarrow \mathcal{W}_{a} \times \mathcal{W}_{a}, \quad w_{0} \mapsto\left(w_{1}, w_{2}\right)$

The closed-loop system $[P, C]$, given by $(2.1)$, is said to be:

- locally well posed if, and only if, it has the existence and uniqueness properties and 
the operator $H_{P, C}: \mathcal{W} \rightarrow \mathcal{W}_{a} \times \mathcal{W}_{a}, w_{0} \mapsto\left(w_{1}, w_{2}\right)$, is causal;

- globally well posed if, and only if, it is locally well posed and $\operatorname{im} H_{P, C} \subset \mathcal{W}_{e} \times \mathcal{W}_{e}$;

- $\mathcal{W}$-stable if, and only if, it is locally well posed and im $H_{P, C} \subset \mathcal{W} \times \mathcal{W}$;

- regularly well posed if, and only if, it is locally well posed and

$$
\forall w_{0} \in \mathcal{W} \quad\left[\omega_{w_{0}}<\infty \quad \Longrightarrow \quad T_{\omega_{w_{0}}} H_{P, C}\left(w_{0}\right) \notin \mathcal{W} \times \mathcal{W}\right]
$$

If $[P, C]$ is globally well posed, then for each $w_{0} \in \mathcal{W}$ the solution $H_{P, C}\left(w_{0}\right)$ exists on the half line $\mathbb{R}_{+}$. Regular well posedness means that if the closed-loop system has a finite escape time $\omega>0$ for some disturbance $\left(u_{0}, y_{0}\right) \in \mathcal{W}$, then at least one of the components $u_{1}, u_{2}$ or $y_{1}, y_{2}$ is not a restriction to $[0, \omega)$ of a function in $\mathcal{U}$ or $\mathcal{Y}$, respectively. If $[P, C]$ is regularly well posed and satisfies

$$
\forall w_{0} \in \mathcal{W} \quad\left[\omega_{w_{0}}<\infty \quad \Longrightarrow \quad T_{\omega_{w_{0}}} H_{P, C}\left(w_{0}\right) \in \mathcal{W} \times \mathcal{W}\right]
$$

there does not exist a solution of $[P, C]$ with a finite escape time, and therefore $[P, C]$ is globally well posed. However, global well posedness does not guarantee that each solution belongs to $\mathcal{W} \times \mathcal{W}$; the latter is ensured by $\mathcal{W}$-stability of $[P, C]$. Note also that neither regular nor global well posedness implies the other.

Our main concern will be the situation wherein the closed-loop system $[P, C]$ is generated by a system of (nonlinear) differential equations. In this context, a globally well-posed system is a system with the property of existence and uniqueness of solutions and for which finite-time blow up does not occur: all (forward) solutions have maximal interval of existence $[0, \infty)$. Regular well posedness usually follows from standard existence theory for differential equations when $\mathcal{W}=L^{\infty} \times L^{\infty}$. However, when $\mathcal{W} \neq L^{\infty} \times L^{\infty}$ (in this paper we are primarily interested in $\mathcal{W}=L^{2} \times L^{2}$ ), stronger properties of the underlying differential equations are required. As shall be shown, all closed-loop systems considered in this paper are regularly well posed.

2.3. Graphs and gain-function stability. In our investigation of robustness of stability properties of a closed-loop system, the concept of graphs and gain-function stability will play a central rôle. Corresponding to a plant operator $P$ (respectively, the controller operator $C$ ) is a subset of $\mathcal{W}$, called the graph of the plant $\mathcal{G}_{P}$ (respectively, the controller $\mathcal{G}_{C}$ ), defined as

$$
\mathcal{G}_{P}=\left\{\left(\begin{array}{c}
u \\
P u
\end{array}\right) \mid u \in \mathcal{U}, P u \in \mathcal{Y}\right\} \subset \mathcal{W}, \quad \mathcal{G}_{C}=\left\{\left(\begin{array}{c}
C y \\
y
\end{array}\right) \mid C y \in \mathcal{U}, y \in \mathcal{Y}\right\} \subset \mathcal{W} .
$$

Note that in general $\mathcal{G}_{P}, \mathcal{G}_{C} \neq \mathcal{W}$.

A causal operator $F: \mathcal{X} \rightarrow \mathcal{V}_{a}$ where $\mathcal{X}, \mathcal{V}$ are subsets of normed signal spaces, is said to be gain-function stable (or gf-stable) if, and only if, $\operatorname{im} F \subset \mathcal{V}$ and the following nonlinear so-called gain-function is well defined:

$$
\begin{aligned}
g[F]:\left(r_{0}, \infty\right) & \rightarrow \mathbb{R}_{+}, \\
r & \mapsto g[F](r)=\sup \left\{\left\|T_{\tau} F x\right\| \mid x \in \mathcal{X},\left\|T_{\tau} x\right\| \in\left(r_{0}, r\right], \tau>0\right\},
\end{aligned}
$$

where $r_{0}:=\inf _{x \in \mathcal{X}}\|x\|<\infty$. Observe that $\|F x\|_{\tau} \leq g[F]\left(\|x\|_{\tau}\right)$. A closed-loop system $[P, C]$ is said to be gf-stable if, and only if, it is globally well posed and $H_{P, C}: \mathcal{W} \rightarrow \mathcal{W}_{e} \times \mathcal{W}_{e}$ is gf-stable.

Note the following facts: (i) global well posedness of $[P, C]$ implies that im $H_{P, C} \subset$ $\mathcal{W}_{e} \times \mathcal{W}_{e}$; (ii) gain function stability of $[P, C]$ implies $\mathcal{W}$-stability of $[P, C]$; (iii) if 
$[P, C]$ is $\mathcal{W}$-stable, then $H_{P, C}: \mathcal{W} \rightarrow \mathcal{G}_{P} \times \mathcal{G}_{C}$ is a bijective operator with inverse $H_{P, C}^{-1}:\left(w_{1}, w_{2}\right) \mapsto w_{1}+w_{2}$. To see (iii), note that im $H_{P, C} \subset \mathcal{W} \times \mathcal{W}$ implies that $\operatorname{im} H_{P, C} \subset \mathcal{G}_{P} \times \mathcal{G}_{C}$, and since for any $w_{1} \in \mathcal{G}_{P} \subset \mathcal{W}, w_{2} \in \mathcal{G}_{C} \subset \mathcal{W}$ we have $w_{1}+w_{2} \in \mathcal{W}$, it follows that $\operatorname{im} H_{P, C} \supset \mathcal{G}_{P} \times \mathcal{G}_{C}$. Therefore, we can think of an gf-stable $H_{P, C}$ as a surjective operator $H_{P, C}: \mathcal{W} \rightarrow \mathcal{G}_{P} \times \mathcal{G}_{C}$. The inverse of $H_{P, C}: \mathcal{W} \rightarrow \mathcal{G}_{P} \times \mathcal{G}_{C}$ is obviously $H_{P, C}^{-1}:\left(w_{1}, w_{2}\right) \mapsto w_{1}+w_{2}$.

Finally, with a closed-loop system $[P, C]$, we associate the following two parallel projection operators: $\Pi_{P / / C}: \mathcal{W} \rightarrow \mathcal{W}_{a}, w_{0} \mapsto w_{1}$, and $\Pi_{C / / P}: \mathcal{W} \rightarrow \mathcal{W}_{a}, w_{0} \mapsto w_{2}$. Clearly, $H_{P, C}=\left(\Pi_{P / / C}, \Pi_{C / / P}\right)$ and $\Pi_{P / / C}+\Pi_{C / / P}=I$. Therefore, gf-stability of one of the operators $\Pi_{P / / C}$ and $\Pi_{C / / P}$ implies the gf-stability of the other, and so gf-stability of either operator implies gf-stability of the closed-loop system $[P, C]$.

2.4. The nonlinear gap. The essence of the paper is an investigation (in a specific adaptive control context) of the persistence, or otherwise, of stability properties of a globally well-posed closed-loop system $[P, C]$ under "sufficiently small" perturbations of the plant or, in other words, when the plant $P$ is replaced by any plant sufficiently "close" to $P$. Here, plants $P_{1}$ and $P_{2}$ are deemed close if, and only if, their respective graphs are close in the gap sense of [3], outlined next.

Let $\Gamma:=\left\{P: \mathcal{U}_{a} \rightarrow \mathcal{Y}_{a} \mid P\right.$ is causal $\}$ and, for $P_{1}, P_{2} \in \Gamma$, define the (possibly empty) set $\mathcal{O}_{P_{1}, P_{2}}:=\left\{\Phi: \mathcal{G}_{P_{1}} \rightarrow \mathcal{G}_{P_{2}} \mid \Phi\right.$ is causal, bijective, and $\left.\Phi(0)=0\right\}$. Write

$$
\vec{\delta}\left(P_{1}, P_{2}\right):=\inf _{\Phi \in \mathcal{O}_{P_{1}, P_{2}}} \sup _{x \in \mathcal{G}_{P_{1}} \backslash\{0\}, \tau>0}\left(\frac{\left\|\left.(\Phi-I)\right|_{\mathcal{G}_{P_{1}}} x\right\|_{\tau}}{\|x\|_{\tau}}\right),
$$

with the convention that $\vec{\delta}\left(P_{1}, P_{2}\right):=\infty$ if $\mathcal{O}_{P_{1}, P_{2}}=\emptyset$. The nonlinear gap $\delta$ is

$$
\delta: \Gamma \times \Gamma \rightarrow[0, \infty], \quad\left(P_{1}, P_{2}\right) \mapsto \delta\left(P_{1}, P_{2}\right):=\max \left\{\vec{\delta}\left(P_{1}, P_{2}\right), \vec{\delta}\left(P_{2}, P_{1}\right)\right\} .
$$

which provides a generalisation of the standard definition of the linear gap $\delta_{0}$ (previously discussed briefly in the Introduction). To explain this, some notation is needed. For $q, m \in \mathbb{N}$, let $R_{q, m}$ denote the set of proper, rational, $(q \times m)$-matrix-valued functions and let $H_{q, m}^{\infty}$ denote the set of analytic and bounded $\mathbb{C}^{q \times m}$-valued functions on the open right half plane $\mathbb{C}_{+}:=\{\lambda \in \mathbb{C} \mid \operatorname{Re}(\lambda)>0\}$. By $R H_{q, m}^{\infty}$, we denote the class of functions in $R_{q, m}$ that are analytic in $\mathbb{C}_{+}$. It is known, see for example [8, pp. 74-75, 261-262], that any $P \in R_{q, m}$ has a normalised right co-prime factorization, that is, $P=N D^{-1}$, where $N \in R H_{q, m}^{\infty}, D \in R H_{m, m}^{\infty}, D$ has an inverse in $R_{m, m}$, and $N^{*} N+D^{*} D=I_{m}$, where $N^{*}(s):=\overline{N(-\bar{s})}^{T}$. Let $\mathcal{U}=L^{2}\left(\mathbb{R}_{+}, \mathbb{R}^{m}\right)$, $\mathcal{Y}=L^{2}\left(\mathbb{R}_{+}, \mathbb{R}^{q}\right)$ for some $m, q \in \mathbb{N}$ and associate, with $\hat{P} \in R_{q, m}$, the linear operator $P: \mathcal{U}_{e} \rightarrow \mathcal{Y}_{e}, u \mapsto y:=\mathbf{L}^{-1}(\hat{P}) \star u$, where $\mathbf{L}$ denotes the Laplace transform and $\star$ denotes convolution. We refer to $P$ as a linear plant with associated transfer function $\hat{P} \in R_{q, m}$. For $i=1,2$, let $P_{i}: \mathcal{U}_{e} \rightarrow \mathcal{Y}_{e}$ be linear plants with associated strictly proper rational transfer functions $\hat{P}_{i}=N_{i} D_{i}^{-1}$, where $\left(N_{i}, D_{i}\right) \in R H_{q, m}^{\infty} \times R H_{m, m}^{\infty}$ are right co-prime factors (analogous to (1.2)), and let $\Pi_{i}: \mathcal{U}_{e} \times \mathcal{Y}_{e} \rightarrow \mathcal{G}_{P_{i}}$ denote the orthogonal projection onto the closed subspace $\mathcal{G}_{P_{i}}$, respectively. The linear gap between these plants is defined as in (1.3), with

$$
\begin{aligned}
\vec{\delta}_{0}\left(\hat{P}_{i}, \hat{P}_{j}\right):=\inf \left\{\left\|\left(\Delta_{N}, \Delta_{D}\right)\right\|_{H^{\infty}} \mid \Delta_{N} \in R H_{q, m}^{\infty}, \Delta_{D} \in R H_{m, m}^{\infty},\right. \\
\left.\hat{P}_{j}=\left(N_{i}+\Delta_{N}\right)\left(D_{i}+\Delta_{D}\right)^{-1}\right\} .
\end{aligned}
$$


In [3, Proposition 5] it is shown that if $\max \left\{\left\|\left(\Pi_{2}-\Pi_{1}\right) \Pi_{1}\right\|,\left\|\left(\Pi_{1}-\Pi_{2}\right) \Pi_{2}\right\|\right\}<1$, then $\vec{\delta}\left(P_{1}, P_{2}\right)=\left\|\left(\Pi_{2}-\Pi_{1}\right) \Pi_{1}\right\|$, and in [2, Lemma 2$]$ it is shown that $\left\|\left(\Pi_{2}-\Pi_{1}\right) \Pi_{1}\right\|=$ $\vec{\delta}_{0}\left(\hat{P}_{1}, \hat{P}_{2}\right)$ if $\vec{\delta}_{0}\left(\hat{P}_{1}, \hat{P}_{2}\right)<1$, the conjunction of which yields

$$
\vec{\delta}\left(P_{1}, P_{2}\right)=\vec{\delta}_{0}\left(\hat{P}_{1}, \hat{P}_{2}\right), \quad \text { if } \delta_{0}\left(\hat{P}_{1}, \hat{P}_{2}\right)<1 .
$$

The topology induced on $R_{q, m}$ by the gap $\delta_{0}$ is called the graph topology [8, p. 235]; note that the graph topology on $\Gamma$ induces the graph topology on $R_{q, m}$ via the subset topology and the Laplace transform $\mathbf{L}$.

3. System classes and the adaptive controller. We are interested in the control of linear $m$-input $m$-output stabilisable $n$-dimensional state space realisations of transfer functions in $R_{m, m}$, i.e. systems of the form

$$
\dot{x}(t)=A x(t)+B u_{1}(t), \quad x(0) \in \mathbb{R}^{n}, \quad y_{1}(t)=C x(t) .
$$

Henceforth, we fix (arbitrarily) the number $m \geq 1$ of inputs/outputs but allow for variation of the state space dimension $n$. We denote this system class by

$$
\mathcal{P}_{n}=\left\{(A, B, C) \in E_{n} \mid n \geq m,(A, B, C) \text { is stabilizable and detectable }\right\}
$$

where $E_{n}:=\mathbb{R}^{n \times n} \times \mathbb{R}^{n \times m} \times \mathbb{R}^{m \times n}$. We also define the subclass of minimum-phase systems with "high-frequency gain" $C B$ having spectrum in $\mathbb{C}_{+}$,

$$
\widetilde{\mathcal{M}}_{n}=\left\{(A, B, C) \in \mathcal{P}_{n} \mid \quad \sigma(C B) \subset \mathbb{C}_{+}, \quad \operatorname{det}\left[\begin{array}{cc}
s I_{n}-A & B \\
C & 0
\end{array}\right] \neq 0 \forall s \in \overline{\mathbb{C}}_{+}\right\} .
$$

Observe that, for each $(A, B, C) \in \widetilde{\mathcal{M}}_{n}$, there exists an element of its similarity orbit $\left\{\left(T A T^{-1}, T B, C T^{-1}\right) \mid T \in \mathbb{R}^{n \times n}\right.$ invertible $\}$ such that

$$
T A T^{-1}=\left[\begin{array}{cc}
A_{1} & A_{2} \\
A_{3} & A_{4}
\end{array}\right], \quad T B=\left[\begin{array}{c}
0 \\
B_{2}
\end{array}\right]=\left[\begin{array}{c}
0 \\
C B
\end{array}\right], \quad C T^{-1}=\left[\begin{array}{ll}
0 & I
\end{array}\right]
$$

where $\sigma\left(B_{2}\right) \subset \mathbb{C}_{+}$and, by the minimum-phase property, $A_{1}$ has spectrum in the open left half complex plane $\mathbb{C}_{-}$. Therefore, we introduce

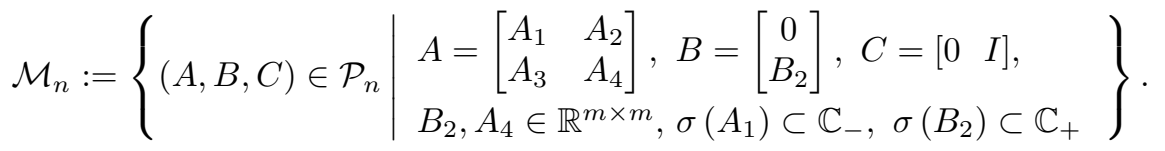

For a system of class $\mathcal{M}_{n},(3.1)$ may be expressed in the equivalent form

$$
\left.\begin{array}{ll}
\dot{z}(t)=A_{1} z(t)+A_{2} y_{1}(t), & z(0)=z^{0} \in \mathbb{R}^{n-m} \\
\dot{y_{1}}(t)=A_{3} z(t)+A_{4} y_{1}(t)+B_{2} u_{1}(t), & y_{1}(0)=y_{1}^{0} \in \mathbb{R}^{m}
\end{array}\right\}, \quad\left(z^{0}, y_{1}^{0}\right)=x^{0} .
$$

We will have occasion to identify $\mathcal{P}_{n}$ with a subspace of Euclidean space $\mathbb{R}^{n^{2}+2 m n}$ by identifying a plant $\theta=(A, B, C)$ with a vector $\theta$ consisting of the elements of the plant matrices, ordered lexigraphically. With normed signal spaces $\mathcal{U}$ and $\mathcal{Y}$ and $\left(\theta, x^{0}\right) \in \mathcal{P}_{n} \times \mathbb{R}^{n}$, we associate the causal plant operator

$$
\widetilde{P}\left(\theta, x^{0}\right): \mathcal{U}_{a} \rightarrow \mathcal{Y}_{a}, \quad u_{1} \mapsto \widetilde{P}\left(\theta, x^{0}\right)\left(u_{1}\right):=y_{1},
$$

where, for $u_{1} \in \mathcal{U}_{a}$ with $\operatorname{dom}\left(u_{1}\right)=[0, \omega)$, we have $y_{1}=C x, x$ being the unique solution of $(3.1)$ on $[0, \omega)$. Note that $\widetilde{P}$ is a map from $\bigcup_{n \geq m}\left(\mathcal{P}_{n} \times \mathbb{R}^{n}\right)$ to the space 
of maps $\mathcal{U}_{a} \rightarrow \mathcal{Y}_{a}$. Furthermore, for $\left(\theta, x^{0}\right)=\left(A, B, C, x^{0}\right) \in \mathcal{P}_{n} \times \mathbb{R}^{n}, \widetilde{P}\left(\theta, x^{0}\right)$ corresponds to a stabilizable and detectable realisation of $C\left(s I_{n}-A\right)^{-1} B \in R_{m, m}$.

Our objective is to study, in the context of systems of form (3.1), the common adaptive strategy

$$
u_{2}(t)=-k(t) y_{2}(t), \quad \dot{k}(t)=\left\|y_{2}(t)\right\|^{2}, \quad k(0)=k^{0} \in \mathbb{R}_{+},
$$

with the associated control operator, parameterized by $k^{0}$, denoted by

$$
\widetilde{C}\left(k^{0}\right): \mathcal{Y}_{a} \rightarrow \mathcal{U}_{a}, \quad y_{2} \mapsto \widetilde{C}\left(k^{0}\right)\left(y_{2}\right):=u_{2} .
$$

Note that $\widetilde{C}$ is a map from $\mathbb{R}_{+}$to the space of causal maps $\mathcal{Y}_{a} \rightarrow \mathcal{U}_{a}$.

In particular and with reference to Figure 2.1, we will study properties of the closed-loop system $\left[\widetilde{P}\left(\theta, x^{0}\right), \widetilde{C}\left(k^{0}\right)\right]$, generated by the application of the adaptive controller (3.8) to system (3.1), in the presence of disturbances $\left(u_{0}, y_{0}\right) \in \mathcal{W}$ satisfying the interconnection equations

$$
u_{0}=u_{1}+u_{2}, \quad y_{0}=y_{1}+y_{2}
$$

Results will be given for systems (3.5) of class (3.4) (such systems will later play the rôle of the nominal plant) and for the more general class of systems (3.1), (3.2) (such systems which will later play the rôle of the perturbed plant).

3.1. Properties of the interconnection of the adaptive controller with the general linear plant. In this section we investigate the interconnection of the adaptive controller (3.7) (with associated operator $\widetilde{C}\left(k^{0}\right)$ ) and any plant in the form (3.1) (with associated operator $\widetilde{P}\left(\theta, x^{0}\right)$ ), where $\left(\theta, x^{0}, k^{0}\right) \in \mathcal{P}_{n} \times \mathbb{R}^{n} \times \mathbb{R}_{+}$.

Proposition 3.1. Let $n \geq m,\left(\theta, x^{0}, k^{0}\right) \in \mathcal{P}_{n} \times \mathbb{R}^{n} \times \mathbb{R}_{+}$and $u_{0}, y_{0} \in$ $L_{\mathrm{loc}}^{\infty}\left(\mathbb{R}_{+}, \mathbb{R}^{m}\right)$. Then the closed-loop initial-value problem given by $(3.1),(3.7)$, (3.9) has the following properties: (i) there exists a unique maximal solution $(x, k):[0, \omega) \rightarrow$ $\mathbb{R}^{n} \times \mathbb{R}_{+}, 0<\omega \leq \infty$; (ii) if $k \in L^{\infty}\left([0, \omega), \mathbb{R}_{+}\right)$, then $\omega=\infty$; (iii) if $y_{2} \in$ $L^{2}\left([0, \omega), \mathbb{R}^{m}\right)$, then $\omega=\infty$.

Proof. (i) follows from the theory of ordinary differential equations.

(ii): If $k \in L^{\infty}\left([0, \omega), \mathbb{R}_{+}\right)$, then consider the following subsystem of the initial-value problem (3.1), (3.7): $\dot{x}(t)=A x(t)+B\left[u_{0}(t)+k(t) y_{2}(t)\right]$. Integration, together with elementary estimates, yields the existence of constants $c_{0}, c_{1}>0$ such that

$$
\|x(t)\| \leq c_{0}\left(e^{c_{1} t}+\int_{0}^{t} e^{c_{1}(t-s)}\left(\left\|u_{0}(s)\right\|+\left\|y_{2}(s)\right\|\right) \mathrm{d} s\right) \quad \forall t \in[0, \omega) .
$$

Suppose $\omega<\infty$. Since $y_{2} \in L^{2}\left([0, \omega), \mathbb{R}^{m}\right)$ (which is equivalent to $k \in L^{\infty}\left([0, \omega), \mathbb{R}_{+}\right)$) and since $u_{0} \in L_{\mathrm{loc}}^{\infty}\left(\mathbb{R}_{+}, \mathbb{R}^{m}\right)$, it follows from the convolution in (3.10) that the right hand side of $(3.10)$ is bounded on $[0, \omega)$ which contradicts maximality of the solution $x$. Therefore, $\omega=\infty$.

(iii): By assumption, $y_{2} \in L^{2}\left([0, \omega), \mathbb{R}^{m}\right)$, and so $t \mapsto k(t)=k^{0}+\left\|y_{2}\right\|_{L^{2}\left([0, t), \mathbb{R}^{m}\right)}^{2}$ is bounded on $[0, \omega)$, and so Assertion (iii) follows from (ii).

Corollary 3.2. Let $\mathcal{U}=\mathcal{Y}=L^{2}\left(\mathbb{R}_{+}, \mathbb{R}^{m}\right), n \geq m,\left(\theta, x^{0}, k^{0}\right) \in \mathcal{P}_{n} \times \mathbb{R}^{n} \times \mathbb{R}_{+}$. Then the closed-loop initial value problem $\left[\widetilde{P}\left(\theta, x^{0}\right), \widetilde{C}\left(k^{0}\right)\right]$ given by $(2.1)$, (3.6) and (3.8) is regularly well posed.

Proof. Let $\mathcal{W}=L^{2}\left(\mathbb{R}_{+}, \mathbb{R}^{m}\right) \times L^{2}\left(\mathbb{R}_{+}, \mathbb{R}^{m}\right)$. The closed-loop $\left[\widetilde{P}\left(\theta, x^{0}\right), \widetilde{C}\left(k^{0}\right)\right]$ is locally well posed by Proposition $3.1(\mathrm{i})$. To prove that $\left[\widetilde{P}\left(\theta, x^{0}\right), \widetilde{C}\left(k^{0}\right)\right]$ is regularly 
well posed, it suffices to show that $(2.2)$ holds. Let $w_{0}=\left(u_{0}, y_{0}\right) \in \mathcal{W}$. Consider $\left(w_{1}, w_{2}\right)=H_{\widetilde{P}\left(\theta, x^{0}\right), \widetilde{C}\left(k^{0}\right)}\left(w_{0}\right)$ where $\operatorname{dom}\left(w_{1}, w_{2}\right)=[0, \omega)$ and is maximal. Suppose $T_{\omega}\left(w_{1}, w_{2}\right) \in \mathcal{W} \times \mathcal{W}$. Then we have $y_{1} \in L^{2}\left([0, \omega), \mathbb{R}^{m}\right)$, which, in view of Proposition 3.1(iii), yields $\omega=\infty$. Hence as $w_{0} \in \mathcal{W}$ is arbitrary, it follows that the closed-loop system is regularly well posed.

3.2. Properties of the interconnection of the adaptive controller with the nominal plant. In this section we consider the closed-loop behaviour of the nominal plant and controller interconnection given by equations (3.5), (3.7), and perturbations $u_{0}, y_{0}$ satisfying (3.9).

Proposition 3.3. Let $n \geq m,(A, B, C) \in \mathcal{M}_{n},\left(x^{0}, k^{0}\right) \in \mathbb{R}^{n} \times \mathbb{R}_{+}$, and $u_{0}, y_{0} \in L^{2}\left([0, \infty), \mathbb{R}^{m}\right)$. Then the closed-loop initial value problem (3.5), (3.7), (3.9) has the following properties: (i) there exists a unique solution $\left(z, y_{1}, k\right):[0, \infty) \rightarrow$ $\mathbb{R}^{n} \times \mathbb{R}_{+}$; (ii) the limit $\lim _{t \rightarrow \infty} k(t)$ exists and is finite; (iii) $u_{1}, y_{1} \in L^{2}\left(\mathbb{R}_{+}, \mathbb{R}^{m}\right)$, $z \in L^{2}\left(\mathbb{R}_{+}, \mathbb{R}^{n-m}\right)$; (iv) $\lim _{t \rightarrow \infty}\left(z(t), y_{1}(t)\right)=0$.

Proof. The closed-loop equations (3.5), (3.7), (3.9) may be expressed as

$$
\begin{aligned}
& \dot{z}(t)=A_{1} z(t)+A_{2} y_{1}(t) \\
& \dot{y_{1}}(t)=A_{3} z(t)+A_{4} y_{1}(t)-B_{2}\left(u_{0}(t)+k(t)\left[y_{0}(t)-y_{1}(t)\right]\right) \\
& \dot{k}(t)=\left\|y_{0}(t)-y_{1}(t)\right\|^{2} \\
& u_{1}(t)=u_{0}(t)+k(t)\left[y_{0}(t)-y_{1}(t)\right] \\
& \left(z(0), y_{1}(0), k(0)\right)=\left(z^{0}, y_{1}^{0}, k^{0}\right) \in \mathbb{R}^{n-m} \times \mathbb{R}^{m} \times \mathbb{R}_{+} .
\end{aligned}
$$

where $x^{0}=\left(z^{0}, y_{1}^{0}\right)$. By Proposition 3.1 there exists a unique maximal solution $\left(z, y_{1}, k\right):[0, \omega) \rightarrow \mathbb{R}^{n} \times \mathbb{R}_{+}$of the initial-value problem (3.11) for some $\omega \in(0, \infty]$. To prove the proposition, we claim that it suffices to show that $y_{1} \in L^{2}\left([0, \omega), \mathbb{R}^{m}\right)$. To argue this claim, assume that $y_{1} \in L^{2}\left([0, \omega), \mathbb{R}^{m}\right)$ and first note that, by Proposition $3.1, \omega=\infty$ and so Assertion (i) holds. Assertion (ii) follows from the third of equations (3.11). Since $u_{0}, y_{0}, y_{1} \in L^{2}\left(\mathbb{R}_{+}, \mathbb{R}^{m}\right)$ and $k$ is bounded, we have $u_{0}+k\left[y_{0}-y_{1}\right]=$ $u_{1} \in L^{2}\left(\mathbb{R}_{+}, \mathbb{R}^{m}\right)$ and, since $\sigma\left(A_{1}\right) \subset \mathbb{C}_{-}$, it follows from the first of equations (3.11) that $z \in L^{2}\left(\mathbb{R}_{+}, \mathbb{R}^{n-m}\right)$ and $z(t) \rightarrow 0$ as $t \rightarrow \infty$, whence Assertion (iii). Finally, writing the second of equations (3.11) in the form

$\dot{y}_{1}(t)=-y_{1}(t)+f(t), \quad f: t \mapsto\left(I+A_{4}\right) y_{1}(t)+A_{3} z(t)-B_{2}\left(u_{0}(t)+k(t)\left[y_{0}(t)-y_{1}(t)\right]\right)$

and noting that $f \in L^{2}\left(\mathbb{R}_{+}, \mathbb{R}^{m}\right)$, we conclude $y_{1}(t) \rightarrow 0$ as $t \rightarrow \infty$ and so (iv) holds.

We proceed to show that $y_{1} \in L^{2}\left([0, \omega), \mathbb{R}^{m}\right)$. First, we assemble some useful inequalities. Recalling that $\sigma\left(A_{1}\right) \subset \mathbb{C}_{-}$, we have $M:=\sup _{t \in \mathbb{R}_{+}}\left\|e^{A_{1} t}\right\|<\infty$ and, by the first of equations (3.11),

$$
\|z(t)\|^{2} \leq M^{2}\left[\left\|z^{0}\right\|^{2}+\left\|A_{2}\right\|^{2}\left\|y_{1}\right\|_{L^{2}\left([0, t), \mathbb{R}^{m}\right)}^{2}\right] \quad \forall t \in[0, \omega) .
$$

Introduce the linear operator

$$
L: L^{2}\left(\mathbb{R}_{+}, \mathbb{R}^{n-m}\right) \rightarrow L^{2}\left(\mathbb{R}_{+}, \mathbb{R}^{n-m}\right), y \mapsto\left(t \mapsto(L v)(t):=\int_{0}^{t} e^{A_{1}(t-\tau)} v(\tau) d \tau\right)
$$

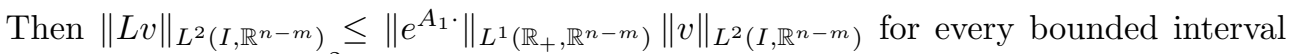
$I \subset \mathbb{R}_{+}$and all $v \in L_{e}^{2}\left(\mathbb{R}_{+}, \mathbb{R}^{n-m}\right)$, which, with the first of equations (3.11), yields

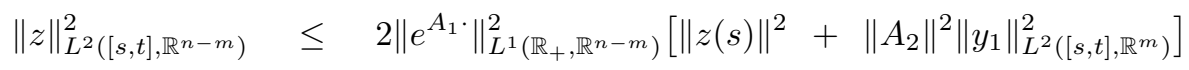


for all $s, t$ with $0 \leq s \leq t<\omega$. Writing

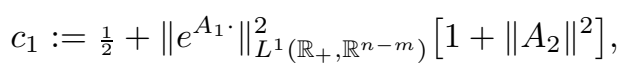

we may infer

$$
\begin{aligned}
\int_{s}^{t}\|z(\tau)\|\left\|y_{1}(\tau)\right\| d \tau \leq \frac{1}{2}\left[\|z\|_{L^{2}\left([s, t], \mathbb{R}^{n-m}\right)}^{2}+\left\|y_{1}\right\|_{L^{2}\left([s, t], \mathbb{R}^{m}\right)}^{2}\right] & \\
\leq c_{1}\left[\|z(s)\|^{2}+\left\|y_{1}\right\|_{L^{2}\left([s, t], \mathbb{R}^{m}\right)}^{2}\right] & \text { for all } s, t \text { with } 0 \leq s \leq t<\omega .
\end{aligned}
$$

Also, observe that, for all $s, t$ with $0 \leq s \leq t<\infty$,

$$
k(t)=k(s)+\left\|y_{0}-y_{1}\right\|_{L^{2}\left([s, t], \mathbb{R}^{m}\right)}^{2} \leq k(s)+\left\|y_{0}\right\|_{L^{2}\left([s, t], \mathbb{R}^{m}\right)}^{2}+\left\|y_{1}\right\|_{L^{2}\left([s, t], \mathbb{R}^{m}\right) .}^{2}
$$

Since $\sigma\left(B_{2}\right) \subset \mathbb{C}_{+}$, the Lyapunov equation $Q B_{2}+B_{2}^{T} Q-2 I=0$ has a unique positive-definite symmetric solution $Q$. From the second of equations (3.11), noting that $\left\|Q B_{2}\right\|=1$ and invoking elementary estimates, we have

$$
\begin{aligned}
\left\langle y_{1}(t), Q \dot{y}_{1}(t)\right\rangle \leq\left\|Q A_{3}\right\|\|z(t)\|\left\|y_{1}(t)\right\|- & \frac{1}{2}\left[k(t)-2\left\|Q A_{4}\right\|-1\right]\left\|y_{1}(t)\right\|^{2} \\
& +\frac{1}{2} k(t)\left\|y_{0}(t)\right\|^{2}+\frac{1}{2}\left\|u_{0}(t)\right\|^{2} \forall t \in[0, \omega)
\end{aligned}
$$

which, on integration, using (3.14), (3.15) and invoking monotonicity of $k$, yields

$$
\begin{aligned}
& 0 \leq\left\langle y_{1}(s), Q y_{1}(s)\right\rangle+2 c_{1}\left\|Q A_{3}\right\|\left(\|z(s)\|^{2}+\left\|y_{1}\right\|_{L^{2}\left([s, t], \mathbb{R}^{m}\right)}^{2}\right) \\
& +\left(k(s)+\left\|y_{0}\right\|_{L^{2}\left([s, t], \mathbb{R}^{m}\right)}^{2}+\left\|y_{1}\right\|_{L^{2}\left([s, t], \mathbb{R}^{m}\right)}^{2}\right)\left\|y_{0}\right\|_{L^{2}\left(\mathbb{R}_{+}, \mathbb{R}^{m}\right)}^{2}+\left\|u_{0}\right\|_{L^{2}\left(\mathbb{R}_{+}, \mathbb{R}^{m}\right)}^{2} \\
& \quad-\left(k(s)-2\left\|Q A_{4}\right\|-1\right)\left\|y_{1}\right\|_{L^{2}\left([s, t], \mathbb{R}^{m}\right)}^{2} \quad \text { for all } s, t \text { with } 0 \leq s \leq t<\omega .
\end{aligned}
$$

Defining

$$
c_{2}:=2 c_{1}\left\|Q A_{3}\right\|+2\left\|Q A_{4}\right\|+2+\left\|y_{0}\right\|_{L^{2}\left(\mathbb{R}_{+}, \mathbb{R}^{m}\right)}^{2},
$$

we have

$$
\begin{array}{r}
0 \leq\left\langle y_{1}(t), Q y_{1}(t)\right\rangle \leq\left\langle y_{1}(s), Q y_{1}(s)\right\rangle+2 c_{1}\left\|Q A_{3}\right\|\|z(s)\|^{2}+\left\|u_{0}\right\|_{L^{2}\left(\mathbb{R}_{+}, \mathbb{R}^{m}\right)}^{2} \\
-\left(k(s)-c_{2}-1\right)\left\|y_{1}\right\|_{L^{2}\left([s, t], \mathbb{R}^{m}\right)}^{2} \text { for all } s, t \text { with } 0 \leq s \leq t<\omega .
\end{array}
$$

Next, observe that

$\left\|y_{1}\right\|_{L^{2}\left([0, t), \mathbb{R}^{m}\right)}^{2} \leq\left\|y_{0}\right\|_{L^{2}\left([0, t), \mathbb{R}^{m}\right)}^{2}+\left\|y_{2}\right\|_{L^{2}\left([0, t), \mathbb{R}^{m}\right)}^{2} \leq\left\|y_{0}\right\|_{L^{2}\left([0, t), \mathbb{R}^{m}\right)}^{2}+k(t) \forall t \in[0, \omega)$.

We consider two possible cases.

Case 1: Assume $k(t) \leq c_{2}$ for all $t \in[0, \omega)$. Then $\left\|y_{1}\right\|_{L^{2}\left([0, \omega), \mathbb{R}^{m}\right)}^{2} \leq\left\|y_{0}\right\|_{L^{2}\left(\mathbb{R}_{+}, \mathbb{R}^{m}\right)}^{2}+c_{2}$.

Case 2: Assume $k(\tau)=c_{2}$ for some $\tau \in[0, \omega)$. Then, by (3.17), we have

$$
\left\|y_{1}\right\|_{L^{2}\left([\tau, \omega), \mathbb{R}^{m}\right)}^{2} \leq\left\langle y_{1}(\tau), Q y_{1}(\tau)\right\rangle+2 c_{1}\left\|Q A_{3}\right\|\|z(\tau)\|^{2}+\left\|u_{0}\right\|_{L^{2}\left(\mathbb{R}_{+}, \mathbb{R}^{m}\right)}^{2} .
$$

By monotonicity, $k(t) \leq c_{2}$ for all $t \in[0, \tau]$ and so $\left\|y_{1}\right\|_{L^{2}\left([0, \tau], \mathbb{R}^{m}\right)}^{2} \leq\left\|y_{0}\right\|_{L^{2}\left(\mathbb{R}_{+}, \mathbb{R}^{m}\right)}^{2}+$ $c_{2}$. Writing

$$
\begin{aligned}
c_{3}:=\left\langle y_{1}^{0}, Q y_{1}^{0}\right\rangle+2 c_{1}\left\|Q A_{3}\right\|\left\|z^{0}\right\|^{2}+\left\|u_{0}\right\|_{L^{2}\left(\mathbb{R}_{+}, \mathbb{R}^{m}\right)}^{2} & +\left(c_{2}+1\right)\left(\left\|y_{0}\right\|_{L^{2}\left(\mathbb{R}_{+}, \mathbb{R}^{m}\right)}^{2}+c_{2}\right),
\end{aligned}
$$


then, by (3.17), it follows that $\left\langle y_{1}(\tau), Q y_{1}(\tau)\right\rangle \leq c_{3}$. By (3.12), we have

$$
\|z(\tau)\|^{2} \leq c_{4}:=M^{2}\left[\left\|z^{0}\right\|^{2}+\left\|A_{2}\right\|\left\|y_{0}\right\|_{L^{2}\left(\mathbb{R}_{+}, \mathbb{R}^{m}\right)}^{2}+c_{2}\left\|A_{2}\right\|\right] .
$$

We may now conclude that

$$
\begin{aligned}
\left\|y_{1}\right\|_{L^{2}\left([0, \tau], \mathbb{R}^{m}\right)}^{2}+\left\|y_{1}\right\|_{L^{2}\left([\tau, \omega), \mathbb{R}^{m}\right)}^{2} & \\
& \leq c_{5}:=c_{2}+c_{3}+2 c_{1} c_{4}\left\|Q A_{3}\right\|+\left\|\left(u_{0}, y_{0}\right)\right\|_{L^{2}\left(\mathbb{R}_{+}, \mathbb{R}^{2 m}\right)}^{2} .
\end{aligned}
$$

Therefore, in each of Cases 1 and 2, we have $\left\|y_{1}\right\|_{L^{2}\left([0, \omega), \mathbb{R}^{m}\right)}^{2} \leq c_{5}$. $\square$

Proposition 3.3 immediately implies the following.

Corollary 3.4. Let $n \geq m, \mathcal{U}=\mathcal{Y}=L^{2}\left(\mathbb{R}_{+}, \mathbb{R}^{m}\right)$ and $\theta=(A, B, C) \in \mathcal{M}_{n}$, $\left(x^{0}, k^{0}\right) \in \mathbb{R}^{n} \times \mathbb{R}_{+}$. Then the closed loop $\left[\widetilde{P}\left(\theta, x^{0}\right), \widetilde{C}\left(k^{0}\right)\right]$ given by (2.1), (3.5), (3.6), (3.8), (3.9) is globally well posed and $(\mathcal{U} \times \mathcal{Y})$-stable.

Proposition 3.5. Let $n \geq m$ and define

$$
\mathcal{D}:=\mathcal{M}_{n} \times \mathbb{R}^{n} \times \mathbb{R}_{+} \times L^{2}\left(\mathbb{R}_{+}, \mathbb{R}^{m}\right) \times L^{2}\left(\mathbb{R}_{+}, \mathbb{R}^{m}\right) .
$$

There exists a continuous map $\nu: \mathcal{D} \rightarrow \mathbb{R}_{+}$such that, for all

$$
d=\left(A, B, C, x^{0}, k^{0}, u_{0}, y_{0}\right) \in \mathcal{D},
$$

the closed-loop system (3.11) is such that $\left\|\left(u_{1}, y_{1}\right)\right\|_{L^{2}\left(\mathbb{R}_{+}, \mathbb{R}^{2 m}\right)} \leq \nu(d)$.

Proof. Observe that the parameters $c_{i}, i=1, \ldots, 5$, defined in (3.13), (3.16), (3.18), (3.19) and (3.20), depend continuously on the data $d=\left(A, B, C, x^{0}, k^{0}, u_{0}, y_{0}\right)$. In particular, the map $\hat{\nu}: d \mapsto \sqrt{c_{5}}$ is continuous. Let $d \in \mathcal{D}$ be arbitrary. Then, by Proposition 3.3 (and recalling (3.20)), we have $\left\|y_{1}\right\|_{L^{2}\left(\mathbb{R}_{+}, \mathbb{R}^{m}\right)} \leq \hat{\nu}(d)$. Now,

$k(t)=k^{0}+\left\|y_{0}-y_{1}\right\|_{L^{2}\left([0, t), \mathbb{R}^{m}\right)}^{2} \leq \nu^{\prime}(d):=k^{0}+2\left\|y_{0}\right\|_{L^{2}\left(\mathbb{R}_{+}, \mathbb{R}^{m}\right)}^{2}+2(\hat{\nu}(d))^{2} \quad \forall t \in \mathbb{R}_{+}$.

Therefore,

$$
\begin{aligned}
\left\|u_{1}\right\|_{L^{2}\left([0, t), \mathbb{R}^{m}\right)} & =\left\|u_{0}+k(t)\left[y_{0}(t)-y_{1}(t)\right]\right\|_{L^{2}\left([0, t), \mathbb{R}^{m}\right)} \\
& \leq \tilde{\nu}(d):=\left\|u_{0}\right\|_{L^{2}\left(\mathbb{R}_{+}, \mathbb{R}^{m}\right)}+\nu^{\prime}(d)\left(\left\|y_{0}\right\|_{L^{2}\left(\mathbb{R}_{+}, \mathbb{R}^{m}\right)}+\hat{\nu}(d)\right) \quad \forall t \in[0, \omega) .
\end{aligned}
$$

We may now infer that $\left\|\left(u_{1}, y_{1}\right)\right\|_{L^{2}\left(\mathbb{R}_{+}, \mathbb{R}^{2 m}\right)} \leq \nu(d):=\sqrt{(\hat{\nu}(d))^{2}+(\tilde{\nu}(d))^{2}}$.

REMARK 3.6. It is worthwhile to note that $\nu(d) \rightarrow \infty$ as the data approaches the boundary of $\mathcal{M}_{n}$ : for example, if some eigenvalues of $A_{1}$ approach the imaginary axis, then $\|L\| \rightarrow \infty$ and so $c_{1}$, given by (3.13), grows unboundedly; if $\left\|B_{2}\right\| \rightarrow 0$, then $\|Q\| \rightarrow \infty$ and so $c_{2}$, given by (3.16), grows unboundedly. Specifically, there exists a bounded sequence $\left(d_{j}\right)$ in $\mathcal{D}$ such that $\nu\left(d_{j}\right) \rightarrow \infty$ as $j \rightarrow \infty$. However, if $\Omega \subset \mathcal{M}_{n}$ is closed and $\left(d_{j}\right)$ is a bounded sequence in $\Omega \times \mathbb{R}^{n} \times \mathbb{R}_{+} \times L^{2}\left(\mathbb{R}_{+}, \mathbb{R}^{m}\right) \times L^{2}\left(\mathbb{R}_{+}, \mathbb{R}^{m}\right) \subset \mathcal{D}$, then $\left(\nu\left(d_{j}\right)\right)$ is bounded.

3.3. Construction of a gain function. To establish gap margin results, we will need to construct augmented plant and controller operators, as in [1].

Reiterating earlier remarks, we may consider $\mathcal{M}_{n}$ to be a subset of the Euclidean space $E_{n}=\mathbb{R}^{n^{2}+2 m n}$, with the standard Euclidean norm, by identifying a plant $\theta=(A, B, C) \in \mathcal{M}_{n}$ with a vector $\theta \in E_{n}$ consisting of the $n^{2}+2 m n$ elements of the plant matrices ordered lexiographically. Note that $0 \notin \mathcal{M}_{n}$. Let $\mathcal{U}=\mathcal{Y}=L^{2}\left(\mathbb{R}_{+}, \mathbb{R}^{m}\right)$ and define $\widetilde{\mathcal{U}}:=\mathbb{R}^{n^{2}+2 m n} \times \mathcal{U}$ and $\widetilde{\mathcal{W}}:=\widetilde{\mathcal{U}} \times \mathcal{Y}$, which can be considered as signal 
spaces by identifying $\theta \in \mathbb{R}^{n^{2}+2 m n}$ with the constant function $t \mapsto \theta$ and endowing $\widetilde{\mathcal{U}}$ with the norm $\|(\theta, u)\|_{\tilde{\mathcal{U}}}:=\sqrt{\|\theta\|^{2}+\|u\|_{L^{2}\left(\mathbb{R}_{+}, \mathbb{R}^{m}\right)}^{2}}$.

For given $\widetilde{P}(\theta, 0)$ as in (3.6), we define the (augmented) plant operator as

$$
P: \widetilde{\mathcal{U}}_{a} \rightarrow \mathcal{Y}_{a}, \quad\left(\theta, u_{1}\right)=\widetilde{u}_{1} \mapsto y_{1}=P\left(\widetilde{u}_{1}\right):=\widetilde{P}(\theta, 0)\left(\widetilde{u}_{1}\right) .
$$

Fix $k^{0} \geq 0$ and define, for $\widetilde{C}\left(k^{0}\right)$ as in (3.8), the (augmented) controller operator as

$$
C: \mathcal{Y}_{a} \rightarrow \widetilde{\mathcal{U}}_{a}, \quad y_{2} \mapsto \widetilde{u}_{2}=C\left(y_{2}\right):=\left(0, \widetilde{C}\left(k^{0}\right)\left(y_{2}\right)\right) .
$$

For each non-empty $\Omega \subset \mathcal{M}_{n}$, define $\mathcal{W}^{\Omega}:=(\Omega \times \mathcal{U}) \times \mathcal{Y}$, and $H_{P, C}^{\Omega}:=\left.H_{P, C}\right|_{\mathcal{W}^{\Omega}}$. It easily follows from Corollary 3.4 that $H_{P, C}^{\Omega}: \mathcal{W}^{\Omega} \rightarrow \widetilde{\mathcal{W}} \times \widetilde{\mathcal{W}}$ is a causal operator for any $\Omega \subset \mathcal{M}_{n}$. We now establish gf-stability.

Proposition 3.7. Let $n \geq m, k^{0} \geq 0$, and assume $\Omega \subset \mathcal{M}_{n}$ is closed. Then, for the closed-loop system $[P, C]$ given by (2.1), (3.22) and (3.23), the operator $H_{P, C}^{\Omega}$ is gf-stable.

Proof. For $\nu: \mathcal{D} \rightarrow \mathbb{R}_{+}$as in Proposition 3.5 we have, for all $\left(\theta, u_{0}, y_{0}\right) \in \mathcal{W}^{\Omega}$,

$$
\begin{aligned}
\left\|H_{P, C}^{\Omega}\left(\theta, u_{0}, y_{0}\right)\right\|_{\widetilde{\mathcal{W}} \times \widetilde{\mathcal{W}}} \leq\left\|\left(\theta, u_{0}, y_{0}\right)\right\|_{\widetilde{\mathcal{W}}}+2\left\|\left(\theta, u_{1}, y_{1}\right)\right\|_{\widetilde{\mathcal{W}}} & \\
& \leq\left\|\left(u_{0}, y_{0}\right)\right\|_{\mathcal{W}}+3\|\theta\|+2 \nu\left(\theta, 0, k^{0}, u_{0}, y_{0}\right),
\end{aligned}
$$

and so, for $r_{0}:=\inf _{w \in \mathcal{W}^{\Omega}}\|w\|_{\widetilde{\mathcal{W}}}$ and $\alpha \in\left(r_{0}, \infty\right)$, closedness of $\Omega$ yields

$$
\gamma(\alpha):=\sup \left\{\begin{array}{ll}
\left\|\left(u_{0}, y_{0}\right)\right\|_{\mathcal{W}}+3\|\theta\|+2 \nu\left(\theta, 0, k^{0}, u_{0}, y_{0}\right) \mid \begin{array}{l}
\left(\theta, u_{0}, y_{0}\right) \in \mathcal{W}^{\Omega}, \\
\left\|\left(\theta, u_{0}, y_{0}\right)\right\|_{\widetilde{\mathcal{W}}} \leq \alpha
\end{array}
\end{array}\right\}<\infty .
$$

Therefore, a gain-function for $H_{P, C}^{\Omega}$ exists, and the proof is complete.

3.4. Robust stability. In Propositions 3.3 and Corollary 3.4 we have established that, for $k^{0} \geq 0,\left(\theta, x^{0}\right) \in \mathcal{M}_{n} \times \mathbb{R}^{n}$ for some $n \geq m$, and $u_{0}, y_{0} \in L^{2}\left(\mathbb{R}_{+}, \mathbb{R}^{m}\right)$, the closed-loop system $\left[\widetilde{P}\left(\theta, x^{0}\right), \widetilde{C}\left(k^{0}\right)\right]$ is globally well posed and has desirable stability properties. The purpose of this section is to determine conditions under which these properties are maintained when the plant $\widetilde{P}\left(\theta, x^{0}\right)$ is perturbed to a plant $\widetilde{P}\left(\theta_{1}, x_{1}^{0}\right)$ where $\left(\theta_{1}, x_{1}^{0}\right) \in \mathcal{P}_{q} \times \mathbb{R}^{q}$ for some $q \geq m$, in particular when $\theta_{1} \notin \mathcal{M}_{q}$. The essence of the main result Theorem 3.8 is that the stability properties persist if (a) the plants $\widetilde{P}\left(\theta_{1}, 0\right)$ and $\widetilde{P}(\theta, 0)$ are sufficiently close (in the gap sense) and (b) the initial data $x_{1}^{0}$ and disturbance $w_{0}=\left(u_{0}, y_{0}\right)$ are sufficiently small.

TheOREM 3.8. Let $m, n, q \in \mathbb{N}$ with $n \geq m, q \geq m, \mathcal{U}=\mathcal{Y}=L^{2}\left(\mathbb{R}_{+}, \mathbb{R}^{m}\right)$, $\mathcal{W}=\mathcal{U} \times \mathcal{Y}$ and $\theta \in \mathcal{M}_{n}$. For $\left(\vartheta, x^{0}\right)$ in $\mathcal{P}_{q} \times \mathbb{R}_{q}$ or $\mathcal{P}_{n} \times \mathbb{R}_{n}$ and $k^{0} \geq 0$, consider $\widetilde{P}\left(\vartheta, x^{0}\right): \mathcal{U}_{a} \rightarrow \mathcal{Y}_{a}$, and $\widetilde{C}\left(k^{0}\right): \mathcal{Y}_{a} \rightarrow \mathcal{U}_{a}$ as defined by (3.6) and (3.8), respectively. There exist a continuous function $\eta: \mathbb{R}_{+} \rightarrow(0, \infty)$ and a function $\lambda: \mathcal{P}_{q} \rightarrow(0, \infty)$ such that the following holds. For all $\left(\theta_{1}, x_{1}^{0}, w_{0}, r\right) \in \mathcal{P}_{q} \times \mathbb{R}^{q} \times \mathcal{W} \times(0, \infty)$,

$$
\left.\begin{array}{l}
\vec{\delta}\left(\widetilde{P}(\theta, 0), \widetilde{P}\left(\theta_{1}, 0\right)\right) \leq \eta(r) \\
\lambda\left(\theta_{1}\right)\left\|x_{1}^{0}\right\|+\left\|w_{0}\right\|_{\mathcal{W}} \leq r
\end{array}\right\} \quad \Longrightarrow \quad H_{\widetilde{P}\left(\theta_{1}, x_{1}^{0}\right), \widetilde{C}\left(k^{0}\right)}\left(w_{0}\right) \in \mathcal{W} \times \mathcal{W} .
$$

REMARK 3.9. In the set-up of Theorem 3.8, if $H_{\widetilde{P}\left(\theta_{1}, x_{1}^{0}\right), \widetilde{C}\left(k^{0}\right)}\left(w_{0}\right) \in \mathcal{W} \times \mathcal{W}$ with $\theta_{1}=(A, B, C) \in \mathcal{P}_{q}$, then the following hold. (i) If $u_{1}, y_{1} \in L^{2}\left(\mathbb{R}_{+}, \mathbb{R}^{m}\right)$, then 
detectability of $(A, C)$ yields that the solution $x$ of (3.1) belongs to $x \in L^{2}\left(\mathbb{R}_{+}, \mathbb{R}^{m}\right)$. Since $x, \dot{x} \in L^{2}\left(\mathbb{R}_{+}, \mathbb{R}^{m}\right)$, it follows that $x(t) \rightarrow 0$ as $t \rightarrow \infty$. Moreover, the monotone controller gain $k$, given by (3.7), converges to a finite limit. (ii) If $u_{0}, y_{0} \in$ $\left(L^{2} \cap L^{\infty}\right)\left(\mathbb{R}_{+}, \mathbb{R}^{m}\right)$, then $u_{1}, y_{1} \in L^{\infty}\left(\mathbb{R}_{+}, \mathbb{R}^{m}\right)$. This follows from the fact that $x \in L^{\infty}\left(\mathbb{R}_{+}, \mathbb{R}^{m}\right)$ by (i), and so $y_{1} \in L^{\infty}\left(\mathbb{R}_{+}, \mathbb{R}^{m}\right)$. Furthermore, $y_{2} \in L^{2}\left(\mathbb{R}_{+}, \mathbb{R}^{m}\right)$ and so $k \in L^{\infty}\left(\mathbb{R}_{+}, \mathbb{R}\right)$, which, by $u_{2}=k y_{2}$, yields $u_{2} \in L^{\infty}\left(\mathbb{R}_{+}, \mathbb{R}^{m}\right)$, whence $u_{1} \in L^{\infty}\left(\mathbb{R}_{+}, \mathbb{R}^{m}\right)$. (iii) If $u_{0}, y_{0} \in L^{2}\left(\mathbb{R}_{+}, \mathbb{R}^{m}\right)$ and $\lim _{t \rightarrow \infty} u_{0}(t)=\lim _{t \rightarrow \infty} y_{0}(t)=0$, then $\lim _{t \rightarrow \infty} u_{1}(t)=0$. This is a consequence of (i) which gives $\lim _{t \rightarrow \infty} y_{1}(t)=0$, and therefore $\lim _{t \rightarrow \infty} y_{2}(t)=0$, which, by invoking $k \in L^{\infty}\left(\mathbb{R}_{+}, \mathbb{R}\right)$, yields $\lim _{t \rightarrow \infty} u_{2}(t)=$ 0 and so $\lim _{t \rightarrow \infty} u_{1}(t)=0$.

Example 3.10. As an illustrative example, we consider Example ii) in the table of the Introduction, where $P$ and $P_{1}$ are specified in the frequency domain by the associated transfer functions

$$
\hat{P}_{1}(s)=\frac{1}{s-\theta}, \quad \hat{P}_{2}(s)=\frac{N(M-s)}{(N+s)(M+s)(s-\theta)}, \quad N, M \geq \varepsilon^{-1}, \varepsilon>0 .
$$

Note that $\hat{P}_{1}$ has a realisation $\widetilde{P}(\theta, 0) \in \mathcal{M}_{1}$ and $\hat{P}_{2}$ has a realization $\widetilde{P}\left(\theta_{1}, 0\right) \in$ $\mathcal{P}_{3} \backslash \mathcal{M}_{3}$. We claim that $\vec{\delta}_{0}\left(\hat{P}_{1}, \hat{P}_{2}\right) \rightarrow 0$ as $\varepsilon \rightarrow 0+$. To prove this assertion, note that

$$
A(s)=\frac{s-\theta}{s+\sqrt{\theta^{2}+1}}, \quad B(s)=\frac{1}{s+\sqrt{\theta^{2}+1}}
$$

satisfy $\hat{P}_{1}(s)=B(s) A(s)^{-1}, A, B \in H^{\infty}$ and $A^{*}(s) A(s)+B^{*}(s) B(s)=I$. Therefore, $A, B$ form a normalised right co-prime factorisation of $\hat{P}_{1}$. Since

$$
\hat{P}_{2}(s)=\frac{B(s)+\Delta_{B}(s)}{A(s)}, \quad \text { where } \quad \Delta_{B}(s)=\left(\frac{N(M-s)}{(s+N)(s+M)}-1\right) B(s),
$$

and $\Delta_{B} \in H^{\infty}$, by (2.5) it suffices to show that

$$
\left\|\left(\begin{array}{c}
0 \\
\Delta_{B}
\end{array}\right)\right\|_{H^{\infty}}=\left\|\left(\frac{N(M-s)}{(s+N)(s+M)}-1\right) B(s)\right\|_{H^{\infty}} \rightarrow 0 \quad \text { as } \varepsilon \rightarrow 0+,
$$

and this follows from a routine calculation. Thus the claim is proved.

To apply Theorem 3.8 to conclude robust stability, it would suffice to show that $\vec{\delta}\left(\hat{P}_{1}, \hat{P}_{2}\right) \rightarrow 0$ as $\varepsilon \rightarrow 0^{+}$. In view of the equivalence (2.5), the latter could be shown by establishing that the directed gap $\vec{\delta}_{0}\left(\hat{P}_{2}, \hat{P}_{1}\right)$ is less than 1 (recall that $\vec{\delta}_{0}\left(\hat{P}_{1}, \hat{P}_{2}\right) \rightarrow 0$ as $\varepsilon \rightarrow 0^{+}$). Alternatively, anticipating Lemma 4.5 , we can adapt the proof of that lemma (to the case wherein $A, B$ are defined as above and $A^{\prime}=A, B^{\prime}=B+\Delta_{B}$, $\left.(V, U):=\left(A^{*}, B^{*}\right)\right)$, and invoke (3.25), to conclude that

$$
\vec{\delta}\left(\widetilde{P}(\theta, 0), \widetilde{P}\left(\theta_{1}, 0\right)\right) \leq\left\|\left(\begin{array}{c}
0 \\
\Delta_{B}
\end{array}\right)\right\| \cdot\left\|\left(A^{*}, B^{*}\right)\right\|_{H^{\infty}}=\left\|\left(\begin{array}{c}
0 \\
\Delta_{B}
\end{array}\right)\right\| \rightarrow 0 \quad \text { as } \varepsilon \rightarrow 0+.
$$

Thus, under the conditions of Theorem 3.8, the controller $\widetilde{C}\left(k^{0}\right): \mathcal{Y}_{a} \rightarrow \mathcal{U}_{a}$ defined by (3.6), (3.8) stabilizes any stabilizable and detectable realisation of $\hat{P}_{2}$. As observed in the Introduction, $\hat{P}_{2}$ is an example of a plant which violates all the classical assumptions of adaptive control.

To prove Theorem 3.8 we need to show how the gain function stability of the augmented closed loop $[P, C]$ as given in (3.22) and (3.23) yields the robustness property 
(3.24) for the unaugmented closed loop $\left[\widetilde{P}\left(\theta_{1}, x_{1}^{0}\right), \widetilde{C}\left(k^{0}\right)\right]$. This follows from the next result which is a direct consequence of Theorems 5.2 and 5.3 in [1].

Theorem 3.11. Let $m, n, q \in \mathbb{N}$ with $n \geq m, q \geq m, \mathcal{U}=\mathcal{Y}=L^{p}\left(\mathbb{R}_{+}, \mathbb{R}^{m}\right)$, $1 \leq p \leq \infty$, and $\mathcal{W}=\mathcal{U} \times \mathcal{Y}$. Let $\widetilde{K}: \mathcal{Y}_{a} \rightarrow \mathcal{U}_{a}$ be causal, consider $\widetilde{P}(\vartheta, 0): \mathcal{U}_{a} \rightarrow \mathcal{Y}_{a}$ defined in (3.6) for $\left(\vartheta, x^{0}\right)$ in $\mathcal{P}_{q} \times \mathbb{R}_{q}$ or $\mathcal{P}_{n} \times \mathbb{R}_{n}$. Assume that $[\widetilde{P}(\vartheta, 0), \widetilde{K}]$ is regularly well posed for all $\vartheta \in \mathcal{P}_{q}$ and let $\Omega \subset \mathcal{M}_{n}$ be closed. Define

$$
\begin{array}{rlrl}
P: \mathcal{P}_{n} \times \mathcal{U}_{a} \rightarrow \mathcal{Y}_{a}, & \left(\vartheta, u_{1}\right) & \mapsto P\left(\vartheta, u_{1}\right) & =\widetilde{P}(\vartheta, 0)\left(u_{1}\right) \\
C: \mathcal{Y}_{a} \rightarrow \mathcal{P}_{n} \times \mathcal{U}_{a}, & y_{2} \mapsto C\left(y_{2}\right) & =\left(0, \widetilde{K}\left(y_{2}\right)\right) .
\end{array}
$$

If $\left.H_{P, C}\right|_{\Omega \times \mathcal{W}}$ is gf-stable and $T_{\tau} \Pi_{P / / C}$ is continuous for all $\tau>0$, then there exist a continuous function $\mu: \mathbb{R}_{+} \times \Omega \rightarrow(0, \infty)$ and a function $\lambda: \mathcal{P}_{q} \rightarrow(0, \infty)$ such that, for all $\left(\theta_{1}, \theta, x_{1}^{0}, w_{0}, r\right) \in \mathcal{P}_{q} \times \Omega \times \mathbb{R}^{q} \times \mathcal{W} \times(0, \infty)$,

$$
\left.\begin{array}{l}
\vec{\delta}\left(\widetilde{P}(\theta, 0), \widetilde{P}\left(\theta_{1}, 0\right)\right) \leq \mu(r, \theta) \\
\lambda\left(\theta_{1}\right)\left\|x_{1}^{0}\right\|+\left\|w_{0}\right\|_{\mathcal{W}} \leq r
\end{array}\right\} \quad \Longrightarrow \quad H_{\widetilde{P}\left(\theta_{1}, x_{1}^{0}\right), \widetilde{K}}\left(w_{0}\right) \in \mathcal{W} \times \mathcal{W} .
$$

We are now in a position to prove Theorem 3.8

Proof. Let $\theta \in \mathcal{M}_{n}$ and define $\Omega=\{\theta\}$. Consider Theorem 3.11 with $p=2$ and $\widetilde{K}=\widetilde{C}\left(k^{0}\right)$, where $\widetilde{C}\left(k^{0}\right)$ is given by (3.8). Note that by Corollary 3.2 , the closed loop $\left[\widetilde{P}(\vartheta, 0), \widetilde{C}\left(k^{0}\right)\right]$ is regularly well posed for all $\vartheta \in \mathcal{P}_{q}$. For $P$ and $C$ as defined in Theorem 3.11, the operator $H_{P, C}^{\Omega}$ is gf-stable by Proposition 3.7. By, for example, the proof of Theorem 4.D in [10], $T_{\tau} \Pi_{\widetilde{P}(\theta, 0) / / \widetilde{C}\left(k^{0}\right)}$ is continuous for all $\tau>0$, and therefore $\left.T_{\tau} \Pi_{P / / C}\right|_{\Omega \times \mathcal{W}}$ is continuous for all $\tau>0$. Now all hypotheses of Theorem 3.11 are in place and so there exist a continuous function $\mu: \mathbb{R}_{+} \times \Omega \rightarrow(0, \infty)$ and a function $\lambda: \mathcal{P}_{q} \rightarrow(0, \infty)$ such that (3.26) holds. Statement (3.24) follows on setting $\eta(\cdot)=\mu(\cdot, \theta)$.

4. Georgiou and Smith's example re-visited. In this section we re-consider Example 9 in the paper by Georgiou and Smith [3] (see also [4]). This serves two purposes: to clarify some of the informal arguments therein in relation to robustness with respect to initial data; to demonstrate that, in the $L^{2}$ setting of the present paper, the robustness bound in Theorem 3.8 is qualitatively tight in the sense that it is necessarily dependent on the data $u_{0}, y_{0}, x_{1}^{0}$.

4.1. The nominal and perturbed closed-loop systems. After appropriate re-scaling and re-labelling of variables, the first-order linear plant $\widetilde{P}\left(a, y_{1}^{0}\right)$, parameterized by $a \in \mathbb{R}$ and $y_{1}^{0} \in \mathbb{R}$, considered in [3, Example 9] can be expressed as

$$
\widetilde{P}\left(a, y_{1}^{0}\right): \mathcal{U}_{a} \rightarrow \mathcal{Y}_{a}, \quad u_{1} \mapsto y_{1}, \quad \text { where } \dot{y}_{1}=a y_{1}+u_{1}, \quad y_{1}(0)=y_{1}^{0},
$$

and so, for $u_{1} \in \mathcal{U}_{a}, y_{1}=\widetilde{P}\left(a, y_{1}^{0}\right)\left(u_{1}\right): \operatorname{dom}\left(u_{1}\right) \rightarrow \mathbb{R}$ is the unique maximal solution of the initial-value problem in (4.1). The controller, parameterized by $k^{0} \in \mathbb{R}_{+}$, is

$$
\widetilde{C}\left(k^{0}\right): \mathcal{Y}_{a} \rightarrow \mathcal{U}_{a}, y_{2} \mapsto u_{2}:=-k y_{2}, \quad \text { where } \dot{k}=y_{2}^{2}, \quad k(0)=k^{0},
$$

and so, for $y_{2} \in \mathcal{Y}_{a},-k y_{2}=u_{2}=\widetilde{C}\left(k^{0}\right)\left(y_{2}\right): \operatorname{dom}\left(y_{2}\right) \rightarrow \mathbb{R}$, where $k$ is the unique maximal solution of the initial-value problem in (4.2)

The closed-loop system $\left[\widetilde{P}\left(a, y_{1}^{0}\right), \widetilde{C}\left(k^{0}\right)\right]$ will be analyzed in the two settings of $\mathcal{U}=\mathcal{Y}=L^{2}\left(\mathbb{R}_{+}, \mathbb{R}\right)$ and $\mathcal{U}=\mathcal{Y}=L^{\infty}\left(\mathbb{R}_{+}, \mathbb{R}\right)$. In view of Proposition 3.1(i), for all 
$a, y_{1}^{0} \in \mathbb{R}$ and $k^{0} \in \mathbb{R}_{+}$, the closed-loop system $\left[\widetilde{P}\left(a, y_{1}^{0}\right), \widetilde{C}\left(k^{0}\right)\right]$ is locally well posed in both settings; moreover, by Proposition 3.3, in the former $L^{2}$ setting, the closed loop system is globally well posed, and the signals $y_{1}$ and $k$ are bounded, with $y_{1}(t) \rightarrow 0$ and $k(t) \rightarrow k^{\infty} \in \mathbb{R}$ as $t \rightarrow \infty$.

As in [3, Example 9], consider a perturbation of the plant $\widetilde{P}\left(a, y_{1}^{0}\right)$ consisting of the series connection of $\frac{1}{s-a}$ (i.e. the transfer function associated with $\left.\widetilde{P}\left(a, y_{1}^{0}\right)\right)$ and an all-pass factor $\frac{M-s}{M+s}$ with $M>0$. As a realisation of this series connection, consider

$$
\dot{y}_{1}=a y_{1}+z-u_{1}, \quad \dot{z}=-M\left[z-2 u_{1}\right], \quad\left(y_{1}(0), z(0)\right)=\left(y_{1}^{0}, z^{0}\right) .
$$

This series connection is denoted by

$$
\widetilde{P}_{1}\left(a, M, y_{1}^{0}, z^{0}\right): \mathcal{U}_{a} \rightarrow \mathcal{Y}_{a}, \quad u_{1} \mapsto y_{1} .
$$

The closed-loop equations for $\left[\widetilde{P}_{1}\left(a, M, y_{1}^{0}, z^{0}\right), \widetilde{C}\left(k^{0}\right)\right]$ are given by

$$
\left.\begin{array}{ll}
\dot{y}_{1}(t)=[a+k(t)] y_{1}(t)+z(t)-k(t) y_{0}(t)-u_{0}(t), & y_{1}(0)=y_{1}^{0}, \\
\dot{z}(t)=-2 M k(t) y_{1}(t)-M z(t)+2 M k(t) y_{0}(t)+2 M u_{0}(t), & z(0)=z^{0}, \\
\dot{k}(t)=\left(y_{0}(t)-y_{1}(t)\right)^{2}, & k(0)=k^{0}, \\
u_{1}(t)=u_{0}(t)+k(t) y_{0}(t)-k(t) y_{1}(t), &
\end{array}\right\}
$$

For fixed (but arbitrary) $a \in \mathbb{R}$ and $k^{0} \in \mathbb{R}_{+}$, and applying Theorem 3.8, we may conclude the existence of a continuous function $\eta: \mathbb{R}_{+} \rightarrow(0, \infty)$ and a function $\lambda_{1}: \mathbb{R} \rightarrow(0, \infty)$ such that, if (i) $\vec{\delta}\left(\widetilde{P}(a, 0), \widetilde{P}_{1}(a, M, 0,0) \leq \eta(r)\right.$ for some $r>0$, then, for all initial data $x_{1}^{0}=\left(y_{1}^{0}, z^{0}\right)$ and all disturbances $u_{0}, y_{0} \in L^{2}\left(\mathbb{R}_{+}, \mathbb{R}\right)$ satisfying (ii) $\lambda_{1}(M)\left\|x_{1}^{0}\right\|+\left\|\left(u_{0}, y_{0}\right)\right\|_{L^{2}\left(\mathbb{R}_{+}, \mathbb{R}^{2}\right)} \leq r$, the closed-loop system is globally well posed and is such that $\left(y_{1}(t), z(t)\right) \rightarrow(0,0)$ as $t \rightarrow \infty$ and the monotone gain $k$ converges to a finite limit. At this point, we briefly digress to prove a technicality which will prove convenient in later analyses.

Lemma 4.1. Let $M>0, a=0, u_{0}, y_{0} \in L^{2}\left(\mathbb{R}_{+}, \mathbb{R}\right) \cup L^{\infty}\left(\mathbb{R}_{+}, \mathbb{R}\right)$ and $\left(y_{1}^{0}, z^{0}, k^{0}\right) \in$ $\mathbb{R} \times \mathbb{R} \times \mathbb{R}_{+}$. Let $\left(y_{1}, z, k\right):[0, \omega) \rightarrow \mathbb{R} \times \mathbb{R} \times \mathbb{R}_{+}$be the unique maximal solution to the closed-loop initial-value problem (4.4). If there exists $T \in[0, \omega)$ such that $k(T) \geq 4 M$, $\left(y_{0}(T)-y_{1}(T), z(T)\right) \neq(0,0)$, and $\left(u_{0}(t), y_{0}(t)\right)=(0,0)$ for all $t \in[T, \infty)$, then (i) $u_{1}, y_{1} \notin L^{\infty}([0, \omega), \mathbb{R})$ and (ii) $k \notin L^{\infty}\left([0, \omega), \mathbb{R}_{+}\right)$.

Proof. Writing $y_{2}:=y_{0}-y_{1}$, then, by the hypotheses,

$$
\left.\begin{array}{l}
\dot{y}_{2}(t)=k(t) y_{2}(t)-z(t), \quad \dot{z}(t)=2 M k(t) y_{2}(t)-M z(t) \\
\dot{k}(t)=\left(y_{2}(t)\right)^{2}
\end{array}\right\} \quad \forall t \in[T, \omega) .
$$

Defining $\eta:=y_{2}-z /(2 M)$, we have

$$
\left.\begin{array}{l}
\dot{y}_{2}(t)=[k(t)-2 M] y_{2}(t)+2 M \eta(t) \\
\dot{\eta}(t)=-M y_{2}(t)+M \eta(t), \quad \dot{k}(t)=\left(y_{2}(t)\right)^{2}
\end{array}\right\} \quad \forall t \in[T, \omega)
$$

Introduce $W:[T, \omega) \rightarrow \mathbb{R}_{+}, t \mapsto \frac{1}{2}\left[y_{2}^{2}+2 \eta^{2}\right](t)$. By hypothesis, $\left(y_{2}(T), z(T)\right) \neq(0,0)$ and so $W(T)>0$. Moreover, since $k(T) \geq 4 M$, we have

$$
\dot{W}(t)=(k(t)-2 M)\left(y_{2}(t)\right)^{2}+2 M(\eta(t))^{2} \geq 2 M W(t) \quad \forall t \in[T, \omega) .
$$

Therefore,

$$
W(t) \geq e^{2 M(t-T)} W(T) \quad \forall t \in[T, \omega), \quad W(T)>0 .
$$


Seeking a contradiction, suppose that $y_{2}$ is bounded on $[T, \omega$ ). Then $\omega=\infty$ (to see this, simply note that, if $\omega$ is finite, then $y_{2}$ is square integrable and so $k$ is bounded, which, together with Proposition 3.1(ii), yields a contradiction). Let $c_{0}>0$ be such that $\left(y_{2}(t)\right)^{2} \leq c_{0}$ for all $t \in[T, \infty)$ and so $0 \leq k(t) \leq k(T)+c_{0}[t-T]$ for all $t \geq 0$. By (4.5), there exists $c_{1}>0$ such that $|z(t)| \leq c_{1}[1+t]$ for all $t \in[T, \infty)$. Hence, there exists $c_{2}>0$ such that $|\eta(t)| \leq c_{2}[1+t]$ for all $t \in[T, \infty)$. Therefore, it follows that $W(t) \leq \frac{1}{2}\left[c_{0}+2 c_{2}^{2}(1+t)^{2}\right]$ for all $t \geq T$, which contradicts (4.7). Therefore, $y_{2}$ is unbounded on $[T, \omega)$ and so, since $y_{1}(t)=-y_{2}(t)$ for all $t \in[T, \omega)$, we have $y_{1} \notin L^{\infty}([0, \omega), \mathbb{R})$. Finally, and again seeking a contradiction, suppose that $k$ is bounded. Then, by Proposition 3.1(ii), $\omega=\infty$. By the third of equations (4.5), $y_{2} \in L^{2}([T, \infty), \mathbb{R})$. By the second of equations (4.5), we may conclude that $z \in L^{2}([T, \infty), \mathbb{R})$ and $z(t) \rightarrow 0$ as $t \rightarrow \infty$. Rewriting the first of equations (4.5) in the form $\dot{y}_{2}(t)=-y_{2}(t)+\zeta(t)$, with $\zeta(t):=[1+k(t)] y_{2}(t)-z(t)$, and noting that $\zeta \in L^{2}\left(\mathbb{R}_{+}, \mathbb{R}\right)$, it follows that $y_{2}(t) \rightarrow 0$ as $t \rightarrow \infty$. Therefore, $W(t) \rightarrow 0$ as $t \rightarrow \infty$, which contradicts (4.7). Therefore, $k$ is unbounded and so property (ii) holds. Since both $k, y_{1}$ are unbounded and $k$ is monotone, and $u_{1}(t)=k(t) y_{1}(t)$ for all $t \geq T$, it follows that $u_{1} \notin L^{\infty}([0, \omega), \mathbb{R})$. Therefore, property (i) holds.

\subsection{Non-robustness with respect to large initial conditions.}

Proposition 4.2. For $M, a, y_{1}^{0}, z^{0}, k^{0} \in \mathbb{R}$ and $u_{0}, y_{0} \in L^{2}\left(\mathbb{R}_{+}, \mathbb{R}\right)$, consider the closed-loop system $\left[\widetilde{P}_{1}\left(M, a, y_{1}^{0}, z^{0}\right), \widetilde{C}\left(k^{0}\right)\right]$ defined by (2.1), (4.4), (4.2), (4.3) in the specific case wherein $M>0, a=z^{0}=k^{0}=0$, and $u_{0}=y_{0}=0$.

There exists $\chi>0$ such that, if $\left(y_{1}^{0}\right)^{2}>\chi$, then the unique maximal solution $\left(y_{1}, z, k\right)$ : $[0, \omega) \rightarrow \mathbb{R}^{2} \times \mathbb{R}_{+}, 0<\omega \leq \infty$, of the closed-loop system $\left[\widetilde{P}_{1}\left(M, 0, y_{1}^{0}, 0\right), \widetilde{C}(0)\right]$ has the following properties: (i) $u_{1}, y_{1} \notin L^{\infty}([0, \omega), \mathbb{R})$, and (ii) $k \notin L^{\infty}\left([0, \omega), \mathbb{R}_{+}\right)$.

Proof. Under the above hypothesis, the initial-value problem (4.4) is given by

$$
\left.\begin{array}{l}
\dot{y}_{1}(t)=k(t) y_{1}(t)+z(t), \quad \dot{z}(t)=-2 M k(t) y_{1}(t)-M z(t), \dot{k}(t)=\left(y_{1}(t)\right)^{2}, \\
\left(y_{1}(0), z(0), k(0)\right)=\left(y_{1}^{0}, 0,0\right)
\end{array}\right\}
$$

Let $\left(y_{1}, z, k\right):[0, \omega) \rightarrow \mathbb{R}^{2} \times \mathbb{R}_{+}$be the unique maximal solution of (4.8) with

$$
\left(y_{1}^{0}\right)^{2}>\chi:=\left(32 M^{2}\left(1+64 M^{2}\right)+4\left(1+8 M^{2}\right)^{2}\right) /\left(1-e^{-4 M}\right)
$$

We will consider separately the two possible cases: (a) $\omega<\infty$, and (b) $\omega=\infty$.

Case (a): Assume $\omega<\infty$. Then, by Proposition 3.1, the monotone function $k$ in unbounded. This, in turn, implies that $y_{1} \notin L^{2}([0, \omega), \mathbb{R})$ and so $y_{1} \notin L^{\infty}([0, \omega), \mathbb{R})$. Therefore, properties (i) and (ii) hold.

Case (b): Now assume $\omega=\infty$. For later convenience, we observe that, by (4.8),

$$
\|z\|_{L^{2}([0, t) ; \mathbb{R})} \leq 2\left\|k y_{1}\right\|_{L^{2}([0, t), \mathbb{R})} \quad \forall t \geq 0 .
$$

First, we will show that $k(1)>4 M$. For contradiction, suppose otherwise. Then, $\left\|y_{1}\right\|_{L^{2}([0,1], \mathbb{R})}^{2}=k(1) \leq 4 M$ and, by monotonicity of $k, k(t) \in[0,4 M]$ for all $t \in[0,1]$. From (4.10), it now follows that

$$
\left\|y_{1}\right\|_{L^{2}([0,1], \mathbb{R})}^{2}+\|z\|_{L^{2}([0,1] ; \mathbb{R})}^{2} \leq 4 M\left[1+64 M^{2}\right] .
$$


Define $V:[0,1] \rightarrow \mathbb{R}_{+}, t \mapsto \frac{1}{2}\left(y_{1}^{2}+z^{2}\right)(t)$. Then,

$$
\begin{aligned}
\dot{V}(t) & =k(t)\left(y_{1}(t)\right)^{2}+(1-2 M k(t)) y_{1}(t) z(t)-M(z(t))^{2} \\
& \geq-\left(1+8 M^{2}\right)\left|y_{1}(t) z(t)\right|-M(z(t))^{2} \\
& \geq-2 M(z(t))^{2}-(4 M)^{-1}\left[\left(1+8 M^{2}\right) y_{1}(t)\right]^{2} \\
& \geq-4 M V(t)-(4 M)^{-1}\left[\left(1+8 M^{2}\right) y_{1}(t)\right]^{2} \quad \forall t \in[0,1] .
\end{aligned}
$$

Therefore,

$$
\begin{aligned}
\|V\|_{L^{1}\left([0,1], \mathbb{R}_{+}\right)} & \geq(4 M)^{-1}\left(1-e^{-4 M}\right) V(0)-(4 M)^{-2}\left(1+8 M^{2}\right)^{2}\left\|y_{1}\right\|_{L^{2}([0,1], \mathbb{R})}^{2} \\
& \geq(8 M)^{-1}\left(1-e^{-4 M}\right) \chi-(4 M)^{-1}\left(1+8 M^{2}\right)^{2}>2 M\left(1+64 M^{2}\right),
\end{aligned}
$$

which, in conjunction with (4.11), yields the contradiction

$4 M\left(1+64 M^{2}\right) \geq\left\|y_{1}\right\|_{L^{2}([0,1], \mathbb{R})}^{2}+\|z\|_{L^{2}([0,1], \mathbb{R})}^{2}=2\|V\|_{L^{1}\left([0, \tau], \mathbb{R}_{+}\right)}>4 M\left(1+64 M^{2}\right)$.

Therefore, $k(1)>4 M$. Moreover, since $y_{1}^{0} \neq 0$, we may infer from (4.8), that $\left(y_{1}(1), z(1)\right) \neq(0,0)$. The result follows by application of Lemma 4.1 (with $T=1$ ).

\subsection{Non-robustness with respect to large $\mathrm{L}^{2}$ disturbances.}

Proposition 4.3. For $M, a, y_{1}^{0}, z^{0}, k^{0} \in \mathbb{R}$ and $u_{0}, y_{0} \in L^{2}\left(\mathbb{R}_{+}, \mathbb{R}\right)$, consider the closed-loop system $\left[\widetilde{P}_{1}\left(M, a, y_{1}^{0}, z^{0}\right), \widetilde{C}\left(k^{0}\right)\right]$ defined by (2.1), (4.4), (4.2), (4.3) in the specific case wherein $M>0, a=y_{1}^{0}=z^{0}=k^{0}=0$, and $y_{0}=0$.

There exists $u_{0} \in L^{2}\left(\mathbb{R}_{+}, \mathbb{R}\right)$ such that the unique maximal solution $\left(y_{1}, z, k\right):[0, \omega) \rightarrow$ $\mathbb{R}^{2} \times \mathbb{R}_{+}$of the closed-loop system $\left[\widetilde{P}_{1}(M, 0,0,0), \widetilde{C}(0)\right]$ has the following properties: (i) $y_{1} \notin L^{\infty}([0, \omega), \mathbb{R})$, and (ii) $k \notin L^{\infty}\left([0, \omega), \mathbb{R}_{+}\right)$.

Proof. Let $a=y_{1}^{0}=z^{0}=k^{0}=0, M>0$ and $y_{0}=0$. Fix $r \neq 0$ and denote, by $\left(\tilde{y}_{1}, \tilde{z}, \tilde{k}\right):[0, \tilde{\omega}) \rightarrow \mathbb{R} \times \mathbb{R} \times \mathbb{R}_{+}$, the unique maximal solution of (4.4) with $u_{0}$ given by $u_{0}(t)=r$ for all $t \geq 0$, in which case, we have

$$
\left.\begin{array}{l}
\frac{d}{d t}\left[\tilde{y}_{1}(t)-r\right]=-\left[\tilde{y}_{1}(t)-r\right]+[1+\tilde{k}(t)] \tilde{y}_{1}(t)+[\tilde{z}(t)-2 r], \\
\frac{d}{d t}[\tilde{z}(t)-2 r]=-M[\tilde{z}(t)-2 r]-2 M \tilde{k}(t) \tilde{y}_{1}(t), \\
\frac{d}{d t} \tilde{k}(t)=\left(\tilde{y}_{1}(t)\right)^{2}, \quad\left(\tilde{y}_{1}(0), \tilde{z}(0), \tilde{k}(0)\right)=(0,0,0) .
\end{array}\right\}
$$

For contradiction, suppose that the component $\tilde{k}$ is bounded. Then, by Proposition 3.1, $\tilde{\omega}=\infty$. Since $\tilde{k} \in L^{\infty}\left(\mathbb{R}_{+}\right)$, it follows that $\tilde{y}_{1} \in L^{2}\left(\mathbb{R}_{+}\right)$and so, by the second differential equation in (4.12), we may infer that $\tilde{z}(\cdot)-2 r \in L^{2}\left(\mathbb{R}_{+}\right)$. Noting that $[1+\tilde{k}(\cdot)] \tilde{y}_{1}(\cdot)+[\tilde{z}(\cdot)-2 r] \in L^{2}\left(\mathbb{R}_{+}\right)$, it follows from the first equation in (4.12) that $\tilde{y}_{1}(t) \rightarrow r \neq 0$ as $t \rightarrow \infty$ which contradicts the fact that $\tilde{y}_{1} \in L^{2}\left(\mathbb{R}_{+}\right)$. Therefore, the solution component $\tilde{k}$ is unbounded. Unboundedness of $\tilde{k}$, together with the third differential equation in (4.12), implies the existence of $T \in[0, \tilde{\omega})$ such that $k(T)>4 M$ and $\tilde{y}_{1}(T) \neq 0$. Let $u_{0} \in L^{2}\left(\mathbb{R}_{+}, \mathbb{R}\right)$ be the piecewise constant function $u_{0}:=T_{T} r$ (viz. $u_{0}(t)=r$ on $[0, T)$ and $u_{o}(t)=0$ on $[T, \infty)$ ) and denote, by $\left(y_{1}, z, k\right):[0, \omega) \rightarrow \mathbb{R} \times \mathbb{R} \times \mathbb{R}_{+}$, the unique maximal solution of

$$
\begin{array}{lrl}
\dot{y}_{1}(t)=k(t) y_{1}(t)+z(t)-u_{0}(t), & y_{1}(0) & =0, \\
\dot{z}(t)=-2 M k(t) y_{1}(t)-M z(t)+2 M u_{0}(t), & z(0) & =0, \\
\dot{k}(t)=y_{1}(t)^{2}, & k(0)) & =0 .
\end{array}
$$

Clearly, $\tilde{\omega} \leq \omega$ and $\left(\tilde{y}_{1}(t), \tilde{z}(t), \tilde{k}(t)\right)=\left(y_{1}(t), z(t), k(t)\right)$ for all $t \in[0, \tilde{\omega})$. Therefore, $y_{1}(T)=\tilde{y}_{1}(T) \neq 0$ and $k(T)=\tilde{K}(T)>4 M$. An application of Lemma 4.1 completes the proof. 
4.4. Non-robustness with respect to $\mathbf{L}^{\infty}$ disturbances. The initial calculation in [3, Example 9] (see also [4]), shows that arbitrarily small $L^{\infty}$ disturbances $u_{0}, y_{0} \in L^{\infty}\left(\mathbb{R}_{+}, \mathbb{R}\right)$ can cause $\left\|u_{1}\right\|_{L^{\infty}\left(\mathbb{R}_{+}, \mathbb{R}\right)}$ to be arbitrarily large, and so $H_{P, C}$ is not gf-stable in an $L^{\infty}$ sense, whence the claim therein that the $\left(L^{\infty}\right)$ "robustness margin ... should be assigned the value zero". Note that the gf-stability of $H_{P, C}$ is only a sufficient condition for robust stability. In this context, we next show that any constant non-zero input disturbance (and zero output disturbance) leads to unbounded signals in the perturbed closed loop $\left[\widetilde{P}_{1}\left(a, M, y_{1}^{0}, z^{0}\right), \widetilde{C}\left(k^{0}\right)\right]$. This is not surprising in view of [3, Example 9] where it is shown that the unperturbed closed loop $\left[\widetilde{P}\left(a, y_{1}^{0}\right), \widetilde{C}\left(k^{0}\right)\right]$ is non-robust with respect to $L^{\infty}$ disturbances.

Proposition 4.4. For $M, a, y_{1}^{0}, z^{0}, k^{0} \in \mathbb{R}$ and $u_{0}, y_{0} \in L^{2}\left(\mathbb{R}_{+}, \mathbb{R}\right)$, consider the closed-loop system $\left[\widetilde{P}_{1}\left(M, a, y_{1}^{0}, z^{0}\right), \widetilde{C}\left(k^{0}\right)\right]$ defined by (2.1), (4.4), (4.2), (4.3) in the specific case wherein $M>0, a=y_{1}^{0}=z^{0}=k^{0}=0$, and $y_{0}=0$.

For any $r>0$, there exists $u_{0} \in L^{\infty}\left(\mathbb{R}_{+}, \mathbb{R}\right)$ with $\left\|u_{0}\right\|_{L^{\infty}\left(\mathbb{R}_{+}, \mathbb{R}\right)} \leq r$, such that the unique maximal solution $\left(y_{1}, z, k\right):[0, \omega) \rightarrow \mathbb{R}^{2} \times \mathbb{R}_{+}$of the closed-loop system $\left[\widetilde{P}_{1}(M, 0,0,0), \widetilde{C}(0)\right]$ has following properties: (i) $u_{1}, y_{1} \notin L^{\infty}([0, \omega), \mathbb{R})$, and (ii) $k \notin L^{\infty}\left([0, \omega), \mathbb{R}_{+}\right)$.

Proof. This follows directly from the proof of Proposition 4.3, wherein it was shown that for each, $r>0$, there exists $T \in(0, \omega)$ such that the disturbance $u_{0} \in$ $L^{\infty}\left(\mathbb{R}_{+}, \mathbb{R}\right)$, given by $u_{0}:=T_{T} r$ and with norm $\left\|u_{0}\right\|_{L^{\infty}\left(\mathbb{R}_{+}, \mathbb{R}\right)}=r$, is such that properties (i) and (ii) hold.

Interestingly, our analysis in Sub-sections 4.2, 4.3, 4.4 has not established whether a finite escape time can occur within these closed loops. Simulation evidence presented in [3] makes this a plausible (if not likely), but the question remains open.

4.5. Robustness with respect to small $\mathrm{L}^{2}$ disturbances and small initial conditions. Having noted in Sub-section 4.4 that the $L^{\infty}$ robustness margin should be assigned the value zero, our next task is to show that, in the $L^{2}$ setting of the current paper, the situation is less pessimistic. In the $L^{\infty}$ framework, one last remark is warranted. In [3, Example 9] and based on informal numerical evidence, there is a suggestion that - even with zero disturbances - the closed-loop system fails to be robustly stable if the initial conditions are non-zero. Proposition 4.2 confirms this in the case of large initial conditions. However Proposition 4.6 below subsumes the following observation: with zero disturbances, the closed-loop system is robustly stable for sufficiently small initial conditions.

As noted in [3, Example 8], in the $L^{\infty}$ framework, $\vec{\delta}\left(\widetilde{P}(a, 0), \widetilde{P}_{1}(a, M, 0,0)\right) \rightarrow 0$ as $M \rightarrow \infty$. We now show that this result also holds true in the $L^{2}$ framework.

Lemma 4.5. For $M, a, y_{1}^{0}, z^{0} \in \mathbb{R}$ and $u_{0}, y_{0} \in L^{2}\left(\mathbb{R}_{+}, \mathbb{R}\right)$, consider $\widetilde{P}\left(a, y_{1}^{0}\right)$ and $\widetilde{P}_{1}\left(M, a, y_{1}^{0}, z^{0}\right)$ given by equations (4.1) and (4.3), respectively, in the specific case wherein $M>0$ and $y_{1}^{0}=z^{0}=0$. Then $\vec{\delta}\left(\widetilde{P}(a, 0), \widetilde{P}_{1}(a, M, 0,0)\right) \rightarrow 0$ as $M \rightarrow \infty$.

Proof. It will be convenient to utilize a frequency domain representation of linear operators. Firstly, let $c>0$ and define the rational functions $A, A^{\prime}, B, B^{\prime}$ by $A(s)=A^{\prime}(s):=(s-a) /(s+c), B(s):=1 /(s+c)$, and $B^{\prime}(s):=(M-s) /(s+c)(s+M)$. For $n, m \in \mathbb{N}$, let $H^{2}$ denote the set of all analytic functions $f: \mathbb{C}_{+} \rightarrow \mathbb{C}^{n \times m}$ so that $\int_{\mathbb{R}} \| f\left(\alpha+i \beta \|^{2} \mathrm{~d} \beta\right.$ is finite for all $\alpha>0$. Since, by Paley-Wiener, the extended Laplace transform $\mathbf{L}$ on $L^{2}\left(\mathbb{R}_{+}, \mathbb{R}\right)$ yields an isometric isomorphism between $L^{2}\left(\mathbb{R}_{+}, \mathbb{R}\right)$ and $H^{2}$, we may observe (see [8, p. 234]) that the graphs corresponding to $\widetilde{P}(a, 0)$, $\widetilde{P}_{1}(M, a, 0,0)$ can be written as: $\mathbf{L}\left(\mathcal{G}_{\widetilde{P}}\right)=\left(\begin{array}{c}A \\ B\end{array}\right) H^{2}$, and $\mathbf{L}\left(\mathcal{G}_{\widetilde{P}_{1}}\right)=\left(\begin{array}{l}A^{\prime} \\ B^{\prime}\end{array}\right) H^{2}$. 
Let $(V, U):=(1, c+a)$ and define the mappings $\Phi, \tilde{\Phi}$ :

$$
\begin{gathered}
\tilde{\Phi}: \mathbf{L}\left(\mathcal{G}_{\widetilde{P}(a, 0)}\right) \rightarrow \mathbf{L}\left(\mathcal{G}_{\widetilde{P}_{1}(M, a, 0,0)}\right), \quad\left(\begin{array}{l}
u \\
y
\end{array}\right) \mapsto \tilde{\Phi}\left(\begin{array}{l}
u \\
y
\end{array}\right):=\left(\begin{array}{l}
A^{\prime} \\
B^{\prime}
\end{array}\right)(V, U)\left(\begin{array}{l}
u \\
y
\end{array}\right), \\
\Phi: \mathcal{G}_{\widetilde{P}(a, 0)} \rightarrow \mathcal{G}_{\widetilde{P}_{1}(M, a, 0,0)}, \quad \Phi:=\mathbf{L}^{-1} \tilde{\Phi} \mathbf{L} .
\end{gathered}
$$

Since $(V, U)\left(\begin{array}{l}A \\ B\end{array}\right)=1, A^{\prime}, B^{\prime}$ are co-prime and $\mathbf{L}$ is an isometric isomorphism, it follows that $\Phi \in \mathcal{O}_{\widetilde{P}, \widetilde{P}_{1}}$. Additionally, since every element of $\mathbf{L}\left(\mathcal{G}_{\widetilde{P}}\right)$ is of the form $y=\left(\begin{array}{l}A \\ B\end{array}\right) x$, where $x \in H^{2}$, it follows that

$$
\left.(\tilde{\Phi}-I)\right|_{\mathbf{L}\left(\mathcal{G}_{\tilde{P}}\right)} y=\left(I-\left(\begin{array}{l}
A^{\prime} \\
B^{\prime}
\end{array}\right)(U, V)\right)\left(\begin{array}{l}
A \\
B
\end{array}\right) x=\left(\begin{array}{c}
A-A^{\prime} \\
B-B^{\prime}
\end{array}\right) x=\left(\begin{array}{l}
A-A^{\prime} \\
B-B^{\prime}
\end{array}\right)(V, U) y
$$

and, since $\left.(\Phi-I)\right|_{\mathcal{G}_{\tilde{P}}}=\left.\mathbf{L}^{-1}(\tilde{\Phi}-I)\right|_{\mathbf{L}\left(\mathcal{G}_{\tilde{P}}\right)} \mathbf{L}$, we have

$$
\vec{\delta}\left(\widetilde{P}, \widetilde{P}_{1}\right) \leq\left\|\mathbf{L}^{-1}\left(\left(\begin{array}{c}
A-A^{\prime} \\
B-B^{\prime}
\end{array}\right)(V, U)\right) \mathbf{L}\right\|_{L^{2}}=\left\|\left(\begin{array}{c}
A-A^{\prime} \\
B-B^{\prime}
\end{array}\right)(V, U)\right\|_{H^{\infty}}
$$

Hence,

$$
\vec{\delta}\left(\widetilde{P}, \widetilde{P}_{1}\right) \leq\left\|\left(\begin{array}{c}
0 \\
\frac{2 s}{(s+c)(s+M)}
\end{array}\right)(1, c+a)\right\|_{H^{\infty}} \leq 2 \sqrt{1+(c+a)^{2}}\left\|\frac{s}{(s+c)(s+M)}\right\|_{H^{\infty}} .
$$

A straightforward computation confirms that the right hand side in the above goes to 0 as $M$ tends to $\infty$. This completes the proof.

The final result states that for any disturbance level, $M$ can be chosen to ensure stability of the perturbed closed-loop system; furthermore, this stability is local with respect to initial conditions.

Proposition 4.6. Let $a \in \mathbb{R}$. For any $M>0, y_{1}^{0}, z^{0} \in \mathbb{R}, k^{0}>0$, consider the closed-loop system $\left[\widetilde{P}_{1}\left(M, a, y_{1}^{0}, z^{0}\right), \widetilde{C}\left(k^{0}\right)\right]$ as defined by (2.1), (4.4), (4.3) and (4.2). For any $r>0$ there exists $\hat{M}>0$, and for any $M \geq \hat{M}$ there exists $\varepsilon>0$ such that, if $\left\|\left(y_{1}^{0}, z^{0}\right)\right\| \leq \varepsilon$ and $\left\|\left(u_{0}, y_{0}\right)\right\|_{L^{2}\left(\mathbb{R}_{+}, \mathbb{R}^{2}\right)} \leq r$, then the closed-loop system $\left[\widetilde{P}_{1}\left(M, a, y_{1}^{0}, z^{0}\right), \widetilde{C}\left(k^{0}\right)\right]$ has the following properties: (i) there exists a unique solution $\left(y_{1}, z, k\right): \mathbb{R}_{+} \rightarrow \mathbb{R}^{2} \times \mathbb{R}_{+}$; (ii) $\left(u_{1}, y_{1}\right) \in L^{2}\left(\mathbb{R}_{+}, \mathbb{R}^{2}\right)$; (iii) $\lim _{t \rightarrow \infty}\left(y_{1}(t), z(t)\right)=0$; (iv) $k \in L^{\infty}\left(\mathbb{R}_{+}, \mathbb{R}_{+}\right)$.

Proof. Properties (i), (ii), and (iv) follow from Lemma 4.5 and Theorem 3.8. Therefore, $y_{1}, y_{0}, u_{0}, k y_{0}, k y_{1} \in L^{2}\left(\mathbb{R}_{+}\right)$. Invoking the second differential equation in (4.4), we have $z \in L^{2}\left(\mathbb{R}_{+}\right)$and $\lim _{t \rightarrow \infty} z(t)=0$. By the first equation in (4.4) we have $\dot{y}_{1} \in L^{2}\left(\mathbb{R}_{+}\right)$. It now follows that $\lim _{t \rightarrow \infty} y_{1}(t)=0$, whence Property (iii).

5. Summary. In Sub-section 3.4 we developed a general result establishing a robust stability margin (whose size is dependent on the $L^{2}$ disturbance level and size of the initial condition) for the class of MIMO, relative degree one, non-minimum phase plants whose first Markov parameter lies in the open right-half plane, when controlled by the 'standard' adaptive output feedback controller (1.1).

In Section 4 we have given a qualitative analysis of a first order system $\widetilde{P}\left(0, y_{1}^{0}\right)$ perturbed by an all pass factor $\frac{M-s}{M+s}$ and controlled by a standard adaptive controller as considered by Georgiou and Smith [3, Ex. 9]. The results of Section 4 are summarised in the following table, 


\begin{tabular}{|c|c|c|c|}
\hline $\begin{array}{c}\text { Disturbances } \\
\text { and initial data }\end{array}$ & Stability & $\begin{array}{c}\text { Internal } \\
\text { signals }\end{array}$ & $\begin{array}{c}\text { Controller } \\
\text { gain }\end{array}$ \\
\hline \hline $\begin{array}{c}\text { for any small } L^{2} \text { disturbance }\left(u_{0}, y_{0}\right) \\
\text { and any small initial condition }\left(y_{1}^{0}, z^{0}\right)\end{array}$ & stable & $\left(u_{1}, y_{1}\right) \in L^{2}$ & $k \in L^{\infty}$ \\
\hline there exists large $L^{2}$ disturbance $\left(u_{0}, 0\right)$ & unstable & $\left(u_{1}, y_{1}\right) \notin L^{\infty}$ & $k \notin L^{\infty}$ \\
\hline for any large initial condition $\left(y_{1}^{0}, 0\right)$ & unstable & $\left(u_{1}, y_{1}\right) \notin L^{\infty}$ & $k \notin L^{\infty}$ \\
\hline $\begin{array}{c}\text { there exists an } L^{\infty} \text { disturbance }\left(u_{0}, 0\right) \\
\text { (of any non-zero size) }\end{array}$ & unstable & $\left(u_{1}, y_{1}\right) \notin L^{\infty}$ & $k \notin L^{\infty}$ \\
\hline
\end{tabular}

It is worth noting that the $L^{2}$ analysis in this paper provides a mechanism to prove the stability of the disturbance-free system in the presence of small initial conditions. The informal plausibility arguments presented in [3] for the lack of robustness of closedloop system in the presence of non-zero initial conditions do not predict the stable behaviour of the closed-loop system when the initial conditions are small.

This case study highlights the critical rôle played by the choice of signal space alternative signal spaces may give different robust stability guarantees (in particular, in the disturbance-free case, we have seen that the $L^{\infty}$ analysis does not give any indication of the robustness of the closed-loop system under gap perturbations with non-zero initial conditions, however the $L^{2}$ analysis does establish robustness). This highlights the importance of an $L^{2}$ analysis for considering response to initial conditions. The second and third entries in the above table illustrate that the sufficient conditions for robust stability given by the gap analysis in Section 3.4 cannot be improved qualitatively and emphasize the complementary rôle of initial conditions and disturbances. It is also important to note that zero $L^{\infty}$ robustness margins are not inevitable. An example of an adaptive controller exhibiting a non-zero $L^{\infty}$ margin is given in [1].

\section{REFERENCES}

[1] French, M. (2002): Adaptive control and robustness in the gap metric; Submitted for publication, preprint (revised 2005) available at http://eprints.ecs.soton.ac.uk/10787/

[2] Georgiou, T.T. and Smith, M.C. (1990): Optimal robustness in the gap metric; IEEE Trans. Aut. Control 35(6), 673-686

[3] Georgiou, T.T. and Smith, M.C. (1997): Robustness analysis of nonlinear feedback systems: an input-output approach; IEEE Trans. Aut. Control 42(9), 1200-1221

[4] Georgiou, T.T. and Smith, M.C. (2001): Remarks on "Robustness Analysis of Nonlinear Feedback Systems"; IEEE Trans. Aut. Control 46(1), 171-172

[5] Krstić, M., Kanellakopoulos, I. and Kokotović, P.V. (1995): Nonlinear and Adaptive Control Design, Wiley, New York

[6] Polderman, J.W. and Mareels, I.M.Y. (1996): Adaptive Systems: An Introduction, Birkhäuser, Boston

[7] Rohrs, C., Valavani, L.S., Athans, M. and Stein, G. (1985): Robustness of continuous-time adaptive control algorithms in the presence of unmodeled dynamics; IEEE Trans. Auto. Control 30(9), 881-889

[8] Vidyasagar, M. (1985): Control System Synthesis, MIT Press, Cambridge

[9] Zames, G. and El-Sakkary, A.K. (1980): Unstable systems and feedback: the gap metric; Proc. of the Allerton Conf., 380-385

[10] Zeidler, E. (1986): Nonlinear Functional Analysis and its Applications I: Fixed Point Theorems, Springer, Berlin 


\section{Explanatory Notes}

This section contains the additional material from [1] to underpin Theorem 3.11.

This section is not for publication, but is provided for completeness and ease of reference.

Proof of the quoted Theorem 3.11. Recall that a map $T: X \rightarrow Y$ is said to be compact if and only if i) $T$ is continuous and ii) $T$ maps bounded sets into relatively compact sets.

We first establish the following variant on the gain function stability result of [3]:

Theorem 5.1. Let $\mathcal{U}, \mathcal{Y}$ be signal spaces, and let $\mathcal{W}=\mathcal{U} \times \mathcal{Y}$. Suppose $[P, C]$ is gf-stable and $\left[P_{1}, C\right]$ is regularly well-posed. Let $\mathcal{D} \subset \mathcal{G}_{P}, \mathcal{D}_{1} \subset \mathcal{G}_{P_{1}}, \mathcal{X} \subset \mathcal{W}$ and let $r>0$. Suppose $\Pi_{P / / C} \mathcal{X} \subset \mathcal{D}$ and there exists a causal, gf-stable mapping $\Psi: \mathcal{D} \rightarrow \mathcal{D}_{1}$ such that

(i) $T_{\tau}(I-\Psi) \Pi_{P / / C}: \mathcal{X} \rightarrow \mathcal{W}$ is causal and compact for all $\tau>0$.

(ii) $T_{\tau} w+T_{\tau}(I-\Psi) \Pi_{P / / C} x \in \mathcal{X}$ for all $x, w \in \mathcal{X}, \tau>0$.

(iii) There exists a function $\epsilon(\cdot) \in \mathcal{K}_{\infty}{ }^{1}$ such that

$$
\left\|(I-\Psi) \Pi_{P / / C} x\right\|_{\tau} \leq(1+\epsilon)^{-1}(r), \quad \forall x \in \mathcal{X},\|x\| \leq r, \tau>0 .
$$

Then $\left.H_{P_{1}, C}\right|_{\mathcal{X} \cap B_{r}}: \mathcal{X} \cap B_{r} \rightarrow \mathcal{W} \times \mathcal{W}$ is gf-stable and

$$
\left\|\Pi_{P_{1} / / C} w\right\|_{\tau} \leq g[\Psi] \circ g\left[\Pi_{P / / C}\right] \circ\left(1+\epsilon^{-1}\right)(r) \quad \forall w \in \mathcal{X},\|w\| \leq r, \tau \geq 0 .
$$

Proof. Let $w \in \mathcal{X},\|w\| \leq r$, and let $\left[0, \omega_{w}\right)$ be the maximal interval of existence for $H_{P_{1}, C} w$. Let $\omega_{w}>\tau>0$. Consider the equation

$$
\begin{aligned}
T_{\tau} w & =T_{\tau}\left(I+(\Psi-I) \Pi_{P / / C}\right) x \\
& =T_{\tau}\left(\Pi_{C / / P}+\Psi \Pi_{P / / C}\right) x .
\end{aligned}
$$

We claim that this equation has a solution $x \in V$ where:

$$
V=\left\{x \in \mathcal{X}:\|x\| \leq\left(1+\epsilon^{-1}\right)(r), \exists y \in \mathcal{W} \text { s.t. } x=T_{\tau} y\right\} .
$$

Consider the operator

$$
Q_{w}: V \rightarrow \mathcal{X} \quad: \quad x \mapsto T_{\tau} w+T_{\tau}(I-\Psi) \Pi_{P / / C} x,
$$

where observe, by (ii), that $Q_{w}(V) \subset \mathcal{X}$ as required. By (iii) there exists $\epsilon \in \mathcal{K}_{\infty}$ such that, for all $x \in V$,

$$
\begin{aligned}
\left\|Q_{w} x\right\| & =\left\|T_{\tau} w+T_{\tau}(I-\Psi) \Pi_{P / / C} x\right\| \\
& \leq\|w\|_{\tau}+\left\|(I-\Psi) \Pi_{P / / C} x\right\|_{\tau} \\
& \leq\|w\|_{\tau}+(1+\epsilon)^{-1}\left(\|x\|_{\tau}\right), \\
& \leq r+(1+\epsilon)^{-1} \circ\left(1+\epsilon^{-1}\right)(r), \\
& \leq\left(1+\epsilon^{-1}\right)(r),
\end{aligned}
$$

\footnotetext{
${ }^{1}$ Recall that $\mathcal{K}_{\infty}$ denotes the set of continuous increasing functions $\epsilon:[0, \infty) \rightarrow[0, \infty)$ which satisfy $\epsilon(0)=0$ and $\epsilon(r) \rightarrow \infty$ as $r \rightarrow \infty$.
} 
where the fifth inequality follows from the identity:

$$
r+(1+\epsilon)^{-1} \circ\left(1+\epsilon^{-1}\right)(r)=r+(1+\epsilon)^{-1} \circ(1+\epsilon) \circ \epsilon^{-1}(r)=\left(1+\epsilon^{-1}\right)(r) .
$$

Therefore $Q_{w}(V) \subset V$. Since by (i), $T_{\tau}(I-\Psi) \Pi_{P / / C}$ is compact, it then follows that $Q_{w}$ is compact. Hence by Schauder's fixed point theorem, $Q_{w}$ has a fixed point in $V$. Hence equation (5.3) has a solution $x \in V \subset \mathcal{X}$ as claimed.

Since $\Psi \Pi_{P / / C} x \in \mathcal{G}_{P_{1}}, \Pi_{C / / P} x \in \mathcal{G}_{C}$ and $\Psi, \Pi_{P_{1} / / C}, \Pi_{P / / C}, \Pi_{C / / P}$ are causal, it follows from equation (5.3) that

$$
\begin{aligned}
T_{\tau} \Pi_{P_{1} / / C} w & =T_{\tau} \Pi_{P_{1} / / C} T_{\tau} w \\
& =T_{\tau} \Pi_{P_{1} / / C}\left(T_{\tau} \Pi_{C / / P} x+T_{\tau} \Psi \Pi_{P / / C} x\right) \\
& =T_{\tau} \Pi_{P_{1} / / C}\left(\Pi_{C / / P} x+\Psi \Pi_{P / / C} x\right) \\
& =T_{\tau} \Psi \Pi_{P / / C} x
\end{aligned}
$$

hence since $x \in V$,

$$
\begin{aligned}
\left\|\Pi_{P_{1} / / C} w\right\|_{\tau} & =\left\|\Psi \Pi_{P / / C} x\right\|_{\tau} \\
& \leq g[\Psi] \circ g\left[\Pi_{P / / C}\right]\left(\|x\|_{\tau}\right) \\
& \leq g[\Psi] \circ g\left[\Pi_{P / / C}\right] \circ\left(1+\epsilon^{-1}\right)(r) .
\end{aligned}
$$

As $\mathcal{W}$ has the property that $\sup _{\tau \geq 0}\left\|T_{\tau} x\right\|<\infty$ implies $x \in \mathcal{W}$, and since $\omega_{w}>\tau>0$ was arbitrary it follows that $T_{\omega_{w}} \Pi_{P_{1} / / C} w \in \mathcal{W}$, and so $T_{\omega_{w}} H_{P_{1} / / C} w \in \mathcal{W} \times \mathcal{W}$. Since $\left[P_{1}, C\right]$ is regularly well posed, it follows that $\omega_{w}=\infty$ and $\Pi_{P_{1} / / C} w \in \mathcal{W}$. Since $w \in$ $\mathcal{X} \cap B_{r}$ was arbitrary, it follows that (5.2) holds and hence $H_{P_{1}, C}: \mathcal{X} \cap B_{r} \rightarrow \mathcal{W} \times \mathcal{W}$ is gf-stable as required. This completes the proof. $\square$

Let $\mathcal{R}=R_{2 m, 2 m}$ and recall that $E_{n}=\mathbb{R}^{n^{2}+2 n m}$.

TheOREm 5.2. [3.11] Let $p \in[1, \infty], m, n, q \in \mathbb{N}$ with $n \geq m, q \geq m, \mathcal{U}=\mathcal{Y}=$ $L^{p}\left(\mathbb{R}_{+}, \mathbb{R}^{m}\right)$ and $\mathcal{W}=\mathcal{U} \times \mathcal{Y}$. Let $\widetilde{K}: \mathcal{Y}_{a} \rightarrow \mathcal{U}_{a}$ be causal, consider $\widetilde{P}(\vartheta, 0): \mathcal{U}_{a} \rightarrow \mathcal{Y}_{a}$ defined in (3.6) for $\left(\vartheta, x^{0}\right)$ in $\mathcal{P}_{q} \times \mathbb{R}^{q}$ or $\mathcal{P}_{n} \times \mathbb{R}^{n}$, and suppose $[\widetilde{P}(\vartheta, 0), \widetilde{K}]$ is regularly well posed for all $\vartheta \in \mathcal{P}_{q}$. Define

$$
\begin{array}{rlrl}
P: E_{n} \times \mathcal{U}_{a} \rightarrow \mathcal{Y}_{a}, & \left(\vartheta, u_{1}\right) & \mapsto P\left(\vartheta, u_{1}\right) & =\widetilde{P}(\vartheta, 0)\left(u_{1}\right) \\
C: \mathcal{Y}_{a} \rightarrow E_{n} \times \mathcal{U}_{a}, & y_{2} \mapsto C\left(y_{2}\right) & =\left(0, \widetilde{K}\left(y_{2}\right)\right)^{T} .
\end{array}
$$

Let $\Omega \subset E_{n}$ be closed. Suppose $\left.H_{P, C}\right|_{\Omega \times \mathcal{W}}$ is gf-stable and $T_{\tau} \Pi_{P / / C}$ is continuous for all $\tau>0$. Then there exists a continuous function $\mu: \mathbb{R}_{+} \times \Omega \rightarrow(0, \infty)$ such that for all $\theta \in \Omega, \theta_{1} \in \mathcal{P}_{q}, w_{0} \in \mathcal{W},\left\|w_{0}\right\| \leq r$,

$$
\vec{\delta}\left(\widetilde{P}(\theta, 0), \widetilde{P}\left(\theta_{1}, 0\right)\right) \leq \mu(r, \theta) \quad \Longrightarrow \quad H_{\widetilde{P}\left(\theta_{1}, 0\right), \widetilde{K}} w_{0} \in \mathcal{W} \times \mathcal{W} .
$$

Proof. Let $\epsilon \in \mathcal{K}_{\infty}, 0<\nu<1$ and let $r_{0}=\inf _{\vartheta \in \Omega}|\vartheta|$. Since $[P, C]$ is gf-stable, the gain function $g\left[\left.\Pi_{P / / C}\right|_{\Omega \times \mathcal{W}}\right]:\left(r_{0}, \infty\right) \rightarrow[0, \infty)$ is defined. As $g\left[\left.\Pi_{P / / C}\right|_{\Omega \times \mathcal{W}}\right]$ is monotonically increasing, there exists a continuous function $\gamma:\left(r_{0}, \infty\right) \rightarrow[0, \infty)$ s.t. $\gamma(\alpha) \geq g\left[\left.\Pi_{P / / C}\right|_{\Omega \times \mathcal{W}}\right](\alpha)$ for all $\alpha>r_{0}$. Define continuous functions $\beta:\left(r_{0}, \infty\right) \rightarrow$ $(0, \infty)$ and $\mu: \mathbb{R}_{+} \times \Omega \rightarrow(0, \infty)$ by

$$
\begin{aligned}
\beta(r) & =\min \left\{1-\nu, \inf _{r_{0} \leq \alpha \leq r} \frac{(1+\epsilon)^{-1}(\alpha)}{2 \gamma(\alpha)}\right\}, \\
\mu\left(r_{1}, \vartheta\right) & =\beta\left(\sqrt{r_{1}^{2}+|\vartheta|^{2}}\right) .
\end{aligned}
$$


Observe that $\beta(r)=0$ if and only if $\frac{(1+\epsilon)^{-1}\left(r_{0}\right)}{2 \gamma\left(r_{0}\right)}=0$, which implies $r_{0}=0$. But if $r_{0}=0$, then by the definition of $r_{0}$, and since $\Omega$ is closed, we have $0 \in \Omega \subset \mathcal{P}_{n}$. This is a contradiction, so $r_{0}>0$.

Let $w_{0}=\left(u_{0}, y_{0}\right)^{T} \in \mathcal{W}, \theta \in \Omega \subset \mathcal{P}_{n}, \theta_{1} \in \mathcal{P}_{q}$ be such that

$$
\delta\left(\widetilde{P}(\theta, 0), \widetilde{P}\left(\theta_{1}, 0\right)\right) \leq \mu\left(\left\|w_{0}\right\|, \theta\right) .
$$

Let $r^{2}=2\left(\left\|w_{0}\right\|^{2}+|\theta|^{2}\right)>0$, hence

$$
\delta\left(\widetilde{P}(\theta, 0), \widetilde{P}\left(\theta_{1}, 0\right)\right) \leq \beta(r) .
$$

We need to show $\left(w_{1}, w_{2}\right)=H_{\widetilde{P}\left(\theta_{1}, 0\right), \widetilde{K}}\left(w_{0}\right) \in \mathcal{W} \times \mathcal{W}$.

To apply Theorem 5.1, with the augmented signal space $E_{n} \times \mathcal{W}$ for $\mathcal{W}$, we define

$$
P_{1}: E_{n} \times \mathcal{U}_{a} \rightarrow \mathcal{Y}_{a}, \quad P_{1}\left(\theta_{1}, u_{1}\right)=\widetilde{P}(\theta, 0)\left(u_{1}\right) .
$$
posed.

Since $\left[\widetilde{P}\left(\theta_{1}, 0\right), \widetilde{K}\right]$ is regularly well posed, it follows that $\left[P_{1}, C\right]$ is regularly well

Let

$$
\mathcal{D}=\left\{\left(\begin{array}{c}
\vartheta \\
w
\end{array}\right) \in E_{n} \times \mathcal{W}: w \in \mathcal{G}_{\widetilde{P}(\theta, 0)}, \vartheta=\theta\right\} \subset \mathcal{G}_{P}
$$

and

$$
\mathcal{D}_{1}=\left\{\left(\begin{array}{c}
\vartheta \\
w
\end{array}\right) \in E_{n} \times \mathcal{W}: w \in \mathcal{G}_{\widetilde{P}\left(\theta_{1}, 0\right)}, \vartheta=\theta\right\} \subset \mathcal{G}_{P_{1}}
$$

Let

$$
\mathcal{X}=\left\{\left(\begin{array}{c}
\vartheta \\
w
\end{array}\right) \in E_{n} \times \mathcal{W}: \vartheta=\theta\right\} \subset \Omega \times \mathcal{W} .
$$

We now verify the assumptions of Theorem 5.1. By the definition of $P, C$ and since $\left.\Pi_{P / / C}\right|_{\Omega \times \mathcal{W}}$ is gf-stable, it follows that $\Pi_{P / / C} \mathcal{X} \subset \mathcal{D}$.

We now construct a mapping $\Psi: \mathcal{D} \rightarrow \mathcal{D}_{1}$ with the properties required by Theorem 5.1. First note that

$$
\vec{\delta}\left(\widetilde{P}(\theta, 0), \widetilde{P}\left(\theta_{1}, 0\right)\right)=\inf _{\Phi \in \mathcal{O}} \sup _{w \in \mathcal{G}_{\tilde{P}(\theta, 0)} \backslash\{0\}, \tau>0} \frac{\left\|\left.(I-\Phi)\right|_{\mathcal{G}_{\tilde{P}(\theta, 0)}} w\right\|_{\tau}}{\|w\|_{\tau}},
$$

where

$$
\mathcal{O}=\left\{\Phi: \mathcal{G}_{\widetilde{P}(\theta, 0)} \rightarrow \mathcal{G}_{\widetilde{P}\left(\theta_{1}, 0\right)}: \Phi \text { is causal, bijective and } \Phi(0)=0\right\} .
$$

Hence there exists a mapping $\Phi_{\theta} \in \mathcal{O}$ such that,

$$
2 \vec{\delta}\left(\widetilde{P}(\theta, 0), \widetilde{P}\left(\theta_{1}, 0\right)\right) \geq \sup _{w \in \mathcal{G}_{\tilde{P}(\theta, 0)} \backslash\{0\}, \tau>0} \frac{\left\|\left.\left(I-\Phi_{\theta}\right)\right|_{\mathcal{G}_{\tilde{P}(\theta, 0)}} w\right\|_{\tau}}{\|w\|_{\tau}} .
$$

Furthermore, $\Phi_{\theta}$ can always be chosen to make $\mathbf{L}\left(I-\Phi_{\theta}\right) \in \mathcal{R}$ (in the frequency domain) and strictly proper (see the proof of Proposition 5, [3]), hence $T_{\tau}\left(I-\Phi_{\theta}\right)$ is compact for all $0<\tau<\infty$. 
Define the mapping $\Psi: \mathcal{D} \rightarrow \mathcal{D}_{1}$ by

$$
\Psi\left(\begin{array}{c}
\theta \\
w
\end{array}\right)=\left(\begin{array}{c}
\theta \\
\Phi_{\theta}(w)
\end{array}\right)
$$

We first establish condition (i) of Theorem 5.1. Since the mapping $\Psi$ is causal by the causality of $\Phi_{\theta}$, and since $\left.\Pi_{P / / C}\right|_{\Omega \times \mathcal{W}}$ is gf-stable, hence causal, it follows that $T_{\tau}(I-\Psi) \Pi_{P / / C}: \mathcal{X} \rightarrow \mathcal{W}$ is causal for all $0<\tau<\infty$.

$T_{\tau}\left(I-\Phi_{\theta}\right)$ is continuous hence $T_{\tau}(I-\Psi)$ is continuous for all $0<\tau<\infty$. Since $T_{\tau} \Pi_{P / / C}$ is continuous for all $0<\tau<\infty$ and $\Psi$ is causal, it follows that $T_{\tau}(I-\Psi) \Pi_{P / / C}=T_{\tau}(I-\Psi) T_{\tau} \Pi_{P / / C}$ is continuous for all $0<\tau<\infty$.

As $\left.\Pi_{P / / C}\right|_{\Omega \times \mathcal{W}}$ is gf-stable, $\left.T_{\tau}\left(I-\Phi_{\theta}\right)\right|_{\mathcal{G}_{\tilde{P}(\theta, 0)}}$ is compact, and

$$
\left.(I-\Psi)\right|_{\mathcal{D}}\left(\begin{array}{c}
\theta \\
w
\end{array}\right)=\left(\begin{array}{c}
0 \\
\left(I-\Phi_{\theta}\right)(w)
\end{array}\right), \quad \forall\left(\begin{array}{c}
\theta \\
w
\end{array}\right) \in \mathcal{D},
$$

it follows that $T_{\tau}(I-\Psi) \Pi_{P / / C}: \mathcal{X} \rightarrow \mathcal{W}$ maps bounded sets into relatively compact sets for all $\tau>0$. Hence $T_{\tau}(I-\Psi) \Pi_{P / / C}: \mathcal{X} \rightarrow \mathcal{W}$ is compact for all $\tau>0$ as required.

Condition (ii) of Theorem 5.1 follows from the fact that for all $x, w \in \mathcal{X}$,

$$
T_{\tau} w+T_{\tau}(I-\Psi) \Pi_{P / / C} x=T_{\tau}\left(\begin{array}{c}
\theta \\
w^{\prime}
\end{array}\right) \in \mathcal{X} \quad \text { for some } \quad w^{\prime} \in \mathcal{W}
$$

We now verify the condition (5.1) of Theorem 5.1 to establish condition (iii). First we establish a key inequality:

$$
\begin{aligned}
2 \vec{\delta}\left(\widetilde{P}(\theta, 0), \widetilde{P}\left(\theta_{1}, 0\right)\right) & \geq \sup _{w \in \mathcal{G}_{\tilde{P}(\theta, 0)} \backslash\{0\}, \tau>0} \frac{\left\|\left.\left(I-\Phi_{\theta}\right)\right|_{\mathcal{G}_{\tilde{P}(\theta, 0)}} w\right\|_{\tau}}{\|w\|_{\tau}} \\
& \geq \sup _{(\theta, w) \in \mathcal{D} \backslash\{0\}, \tau>0} \frac{\left\|\left.(I-\Psi)\right|_{\mathcal{D}}\left(\theta, w^{T}\right)^{T}\right\|_{\tau}}{\left\|\left(\theta, w^{T}\right)^{T}\right\|_{\tau}}
\end{aligned}
$$

where the second inequality follows from the equation (5.21).

Now let $\tau>0$ and $x \in \mathcal{X},\|x\| \leq r$. Then since $\Pi_{P / / C} x \in \mathcal{D} \subset \mathcal{G}_{P}$, inequality (5.23) gives:

$$
\begin{aligned}
\left\|(I-\Psi) \Pi_{P / / C} x\right\|_{\tau} & \leq 2 \vec{\delta}\left(\widetilde{P}(\theta, 0), \widetilde{P}\left(\theta_{1}, 0\right)\right)\left\|\Pi_{P / / C} x\right\|_{\tau} \\
& \leq 2 \vec{\delta}\left(\widetilde{P}(\theta, 0), \widetilde{P}\left(\theta_{1}, 0\right)\right) g\left[\left.\Pi_{P / / C}\right|_{\Omega \times \mathcal{W}}\right]\left(\|x\|_{\tau}\right),
\end{aligned}
$$

where the first line follows from inequality (5.23). Since $\|x\|_{\tau} \leq r$, it follows from the definition of $\beta$ and inequality (5.12) that:

$$
\begin{aligned}
\vec{\delta}\left(\widetilde{P}(\theta, 0), \widetilde{P}\left(\theta_{1}, 0\right)\right) g\left[\left.\Pi_{P / / C}\right|_{\Omega \times \mathcal{W}}\right]\left(\|x\|_{\tau}\right) & \leq \beta(r) g\left[\left.\Pi_{P / / C}\right|_{\Omega \times \mathcal{W}}\right]\left(\|x\|_{\tau}\right) \\
& \leq \beta\left(\|x\|_{\tau}\right) g\left[\left.\Pi_{P / / C}\right|_{\Omega \times \mathcal{W}}\right]\left(\|x\|_{\tau}\right) \\
& \leq \beta\left(\|x\|_{\tau}\right) \gamma\left(\|x\|_{\tau}\right) \\
& \leq \frac{1}{2}(1+\epsilon)^{-1}\left(\|x\|_{\tau}\right) .
\end{aligned}
$$

Hence by inequalities (5.24), (5.25), which hold for all $x \in \mathcal{X},\|x\| \leq r$ and $\tau>0$, we have established the inequality (5.1), and hence condition (iii), as required. 
Hence it follows from Theorem 5.1 that $\left.H_{P_{1}, C}\right|_{\mathcal{X} \cap B_{r}}$ is gf-stable and equation (5.2) holds. In particular, since $\left(\begin{array}{c}\theta \\ w_{0}\end{array}\right) \in \mathcal{X},\left(\begin{array}{c}\theta \\ w_{0}\end{array}\right) \in B_{r}$ this implies $H_{P_{1}, C}\left(\begin{array}{c}\theta \\ w_{0}\end{array}\right) \in$ $E_{n} \times \mathcal{W} \times E_{n} \times \mathcal{W}$, and consequently $H_{\widetilde{P}\left(\theta_{1}, 0\right), \widetilde{K}} w_{0} \in \mathcal{W} \times \mathcal{W}$. Since $\theta \in \Omega, w_{0} \in \mathcal{W}$ were arbitrary, the result follows. $\square$

TheOREM 5.3. Let $p \in[1, \infty], m, n \in \mathbb{N}$ with $n \geq m, \mathcal{U}=\mathcal{Y}=L^{p}\left(\mathbb{R}_{+}, \mathbb{R}^{m}\right)$ and $\mathcal{W}=\mathcal{U} \times \mathcal{Y}$. Let $\widetilde{K}: \mathcal{Y}_{a} \rightarrow \mathcal{U}_{a}$ be causal, and consider $\widetilde{P}(\theta, 0): \mathcal{U}_{a} \rightarrow \mathcal{Y}_{a}$ defined in (3.6) where $(\theta, 0) \in \mathcal{P}_{n} \times \mathbb{R}^{n}$. Suppose there exists $r_{0}>0$ such that $H_{\widetilde{P}(\theta, 0), \widetilde{K}} \in \mathcal{W} \times \mathcal{W}$ for all $w_{0} \in \mathcal{W},\left\|w_{0}\right\| \leq r$. Then there exists $\lambda>0$ such that $H_{\widetilde{P}\left(\theta, x_{1}^{0}\right), \widetilde{K}} \in \mathcal{W} \times \mathcal{W}$ for all $\left(\theta, x_{1}^{0}\right) \in \mathcal{P}_{n} \times \mathbb{R}^{n}$ such that $\lambda\left|x_{1}^{0}\right|+\left\|w_{0}\right\| \leq r$.

Proof. Let $\theta_{1}=(A, B, C) \in \mathcal{P}_{q}, x_{1}^{0} \in \mathbb{R}^{q}$. We first characterize the graph $\mathcal{G}_{\widetilde{P}\left(\theta_{1}, x_{1}^{0}\right)}$.

Let $F \in \mathbb{R}^{m \times q}$ be such that $\hat{A}=A+B F$ is Hurwitz (note that a suitable $F$ exists since the system is $\widetilde{P}\left(\theta_{1}, 0\right)$ is stabilizable). Define $N: \mathcal{U} \rightarrow N(\mathcal{U}), v \mapsto u$, $M: \mathcal{U} \rightarrow \mathcal{Y}, v \mapsto y$, where

$$
\begin{aligned}
& \dot{x}=(A+B F) x+B v, \quad x(0)=0 \\
& u=F x+v, \\
& y=C x
\end{aligned}
$$

Observe that $N(\mathcal{U})=V:=\left\{u \in \mathcal{U}: \widetilde{P}\left(\theta_{1}, 0\right) u \in \mathcal{Y}\right\}, N: \mathcal{U} \rightarrow V$ is invertible and $\widetilde{P}\left(\theta_{1}, 0\right)=M N^{-1}$. Let

$$
q_{v}:=\left(\begin{array}{c}
N \\
M
\end{array}\right) v+\left(\begin{array}{c}
F \exp (\hat{A} \cdot) x_{1}^{0} \\
C \exp (\hat{A} \cdot) x_{1}^{0}
\end{array}\right) .
$$

We claim $\mathcal{G}_{\widetilde{P}\left(\theta_{1}, x_{1}^{0}\right)}=Q:=\left\{q_{v} \in \mathcal{W}: v \in \mathcal{U}\right\}$.

Consider any $q_{v} \in Q, v \in \mathcal{U}$. Let $u=N v+F \exp (\hat{A} \cdot) x_{1}^{0}$. Since $N v \in \mathcal{U}, M v \in \mathcal{Y}$ and $\exp (\hat{A} \cdot) \in L^{p}\left[\mathbb{R}, \mathbb{R}^{m}\right]=\mathcal{Y}$, we have $u \in \mathcal{U}$ and,

$$
\begin{aligned}
\widetilde{P}\left(\theta_{1}, x_{1}^{0}\right) u & =\widetilde{P}\left(\theta_{1}, 0\right) N v+\widetilde{P}\left(\theta_{1}, x_{1}^{0}\right)\left(F \exp (\hat{A} \cdot) x_{1}^{0}\right) \\
& =M(N)^{-1} N v+C \exp (\hat{A} \cdot) x_{1}^{0} \\
& =M v+C \exp (\hat{A} \cdot) x_{1}^{0} \in \mathcal{Y}
\end{aligned}
$$

Therefore $q_{v}=\left(u, \widetilde{P}\left(\theta_{1}, x_{1}^{0}\right) u\right)^{T} \in \mathcal{U} \times \mathcal{Y}$, so $q_{v} \in \mathcal{G}_{\widetilde{P}\left(\theta_{1}, x_{1}^{0}\right)}$ and hence $Q \subseteq \mathcal{G}_{\widetilde{P}\left(\theta_{1}, x_{1}^{0}\right)}$.

Conversely suppose $\left(u, \widetilde{P}\left(\theta_{1}, x_{1}^{0}\right) u\right)^{T} \in \mathcal{G}_{\widetilde{P}\left(\theta_{1}, x_{1}^{0}\right)}$. Then

$$
\widetilde{P}\left(\theta_{1}, 0\right)\left(u-F \exp (\hat{A} \cdot) x_{1}^{0}\right)=\widetilde{P}\left(\theta_{1}, x_{1}^{0}\right) u-\widetilde{P}\left(\theta_{1}, x_{1}^{0}\right)\left(F \exp (\hat{A} \cdot) x_{1}^{0}\right)
$$

and since the right hand side lies in $\mathcal{Y}$, it follows that $\widetilde{P}\left(\theta_{1}, 0\right)\left(u-F \exp (\hat{A} \cdot) x_{1}^{0}\right) \in \mathcal{Y}$. Therefore $u-F \exp (\hat{A} \cdot) x_{1}^{0} \in V=\operatorname{im}(N)$, and so there exists $v \in \mathcal{U}$ s.t. $N v=$ $u-F \exp (\hat{A} \cdot) x_{1}^{0}$. Therefore equation (5.28) holds, hence

$$
\left(\begin{array}{c}
u \\
\widetilde{P}\left(\theta_{1}, x_{1}^{0}\right) u
\end{array}\right)=q_{v} \in Q
$$

and so $\mathcal{G}_{\widetilde{P}\left(\theta_{1}, x_{1}^{0}\right)} \subseteq Q$. Therefore we have shown $\mathcal{G}_{\widetilde{P}\left(\theta_{1}, x_{1}^{0}\right)}=Q$ as claimed. 
Now let

$$
\lambda=\left\|\left(\begin{array}{c}
F \exp (\hat{A} \cdot) \\
C \exp (\hat{A} \cdot)
\end{array}\right)\right\|
$$

and suppose $w_{0} \in \mathcal{W}, x_{1}^{0} \in \mathbb{R}^{q}$ satisfy

$$
\lambda\left|x_{1}^{0}\right|+\left\|w_{0}\right\| \leq r .
$$

Then by letting

$$
w_{0}^{\prime}=w_{0}-w_{0}^{\prime \prime}, \quad w_{0}^{\prime \prime}=\left(\begin{array}{c}
F \exp (\hat{A} \cdot) x_{1}^{0} \\
C \exp (\hat{A} \cdot) x_{1}^{0}
\end{array}\right)
$$

we have

$$
\left\|w_{0}^{\prime}\right\| \leq \lambda\left|x_{1}^{0}\right|+\left\|w_{0}\right\| \leq r
$$

hence

$$
H_{\widetilde{P}\left(\theta_{1}, 0\right), \widetilde{K}}\left(w_{0}^{\prime}\right)=\left(w_{1}, w_{2}\right) \in \mathcal{G}_{\widetilde{P}\left(\theta_{1}, 0\right)} \times \mathcal{G}_{\widetilde{K}} .
$$

In particular,

$$
w_{0}^{\prime}=w_{1}+w_{2}
$$

and by rearranging we have

$$
w_{0}=\left(w_{1}+w_{0}^{\prime \prime}\right)+w_{2}
$$

Since $w_{1} \in \mathcal{G}_{\widetilde{P}\left(\theta_{1}, 0\right)}$, there exists $v \in \mathcal{U}$ such that $w_{1}=\left(\begin{array}{c}N \\ M\end{array}\right) v$, hence $w_{1}+w_{0}^{\prime \prime} \in$ $Q=\mathcal{G}_{\widetilde{P}\left(\theta_{1}, x_{1}^{0}\right)}$. Since $w_{2} \in \mathcal{G}_{\widetilde{K}}$,

$$
H_{\widetilde{P}\left(\theta_{1}, x_{1}^{0}\right), \widetilde{K}} w_{0}=\left(w_{1}+w_{0}^{\prime \prime}, w_{2}\right) \in \mathcal{G}_{\widetilde{P}\left(\theta_{1}, x_{1}^{0}\right)} \times \mathcal{G}_{\widetilde{K}} \subset \mathcal{W} \times \mathcal{W}
$$

